\title{
A Density Functional Study of Eight- and Eleven-vertex Polyhedral Borane Structures: Comparison with Bare Germanium Clusters
}

\author{
R. B. King,${ }^{1}$ I. Silaghi-Dumitrescu, ${ }^{2}$ and A. Lupan ${ }^{2}$ \\ Department of Chemistry, University of Georgia, Athens, Georgia, 30602, and Faculty of Chemistry and \\ Chemical Engineering, Babeş-Bolyai University, Cluj-Napoca, Roumania
}

\section{Supporting Information}

Table S1. B/B and B/H connectivities of $\mathrm{B}_{8} \mathrm{H}_{8}{ }^{2-}$

Table S2. B/B and B/H connectivities of $\mathrm{B}_{8} \mathrm{H}_{8}{ }^{4-}$

Table S3. B/B and B/H connectivities of $\mathrm{B}_{11} \mathrm{H}_{11}{ }^{2-}$

Table S4. B/B and B/H connectivities of $\mathrm{B}_{11} \mathrm{H}_{11}^{4-}$

Table S5. B/B and $\mathrm{B} / \mathrm{H}$ connectivities of $\mathrm{B}_{11} \mathrm{H}_{11}{ }^{6-}$

Figure S1. Lowest lying molecular orbitals for isomer $24-1\left(C_{2 v}\right)$ of $\mathrm{B}_{11} \mathrm{H}_{11}{ }^{2-}$.

Figure S2. Lowest lying molecular orbitals for isomer $24-3\left(C_{2 v}\right)$ of $\mathrm{B}_{11} \mathrm{H}_{11}{ }^{2-}$.

\footnotetext{
1 University of Georgia

2 Babes-Bolyai University
} 
Table S1. B/B and B/H Connectivities of $\mathrm{B}_{8} \mathrm{H}_{8}{ }^{2-}$

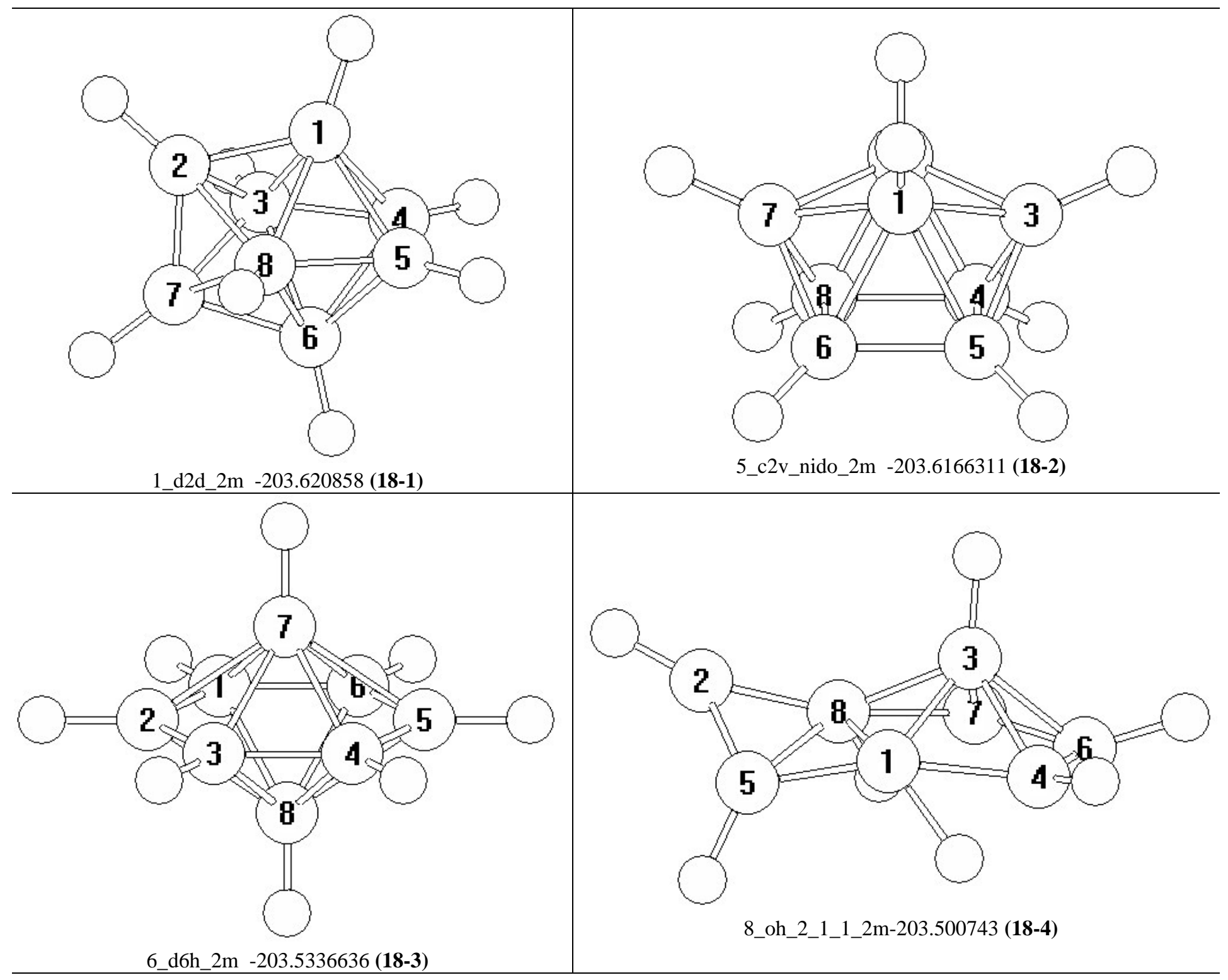




\section{B/B connectivities}

\section{8-1}

\begin{tabular}{|c|c|c|c|c|c|c|c|c|c|}
\hline & 1 & 2 & 3 & 4 & 5 & & 6 & 7 & 8 \\
\hline $1 \mathrm{~B}$ & 0.000000 & & & & & $6 \mathrm{~B}$ & 0.000000 & & \\
\hline $2 \mathrm{~B}$ & 1.614396 & 0.000000 & & & & $7 \mathrm{~B}$ & 1.968675 & 0.000000 & \\
\hline $3 \mathrm{~B}$ & 2.796216 & 1.614396 & 0.000000 & & & $8 \mathrm{~B}$ & 1.968675 & 2.253358 & 0.000000 \\
\hline $4 \mathrm{~B}$ & 3.228792 & 2.796216 & 1.614396 & 0.000000 & & & & & \\
\hline $5 \mathrm{~B}$ & 2.796216 & 3.228792 & 2.796216 & 1.614396 & 0.000000 & & & & \\
\hline $6 \mathrm{~B}$ & 1.614396 & 2.796216 & 3.228792 & 2.796216 & 1.614396 & & & & \\
\hline $7 \mathrm{~B}$ & 1.968675 & 1.968675 & 1.968675 & 1.968675 & 1.968675 & & & & \\
\hline $8 \mathrm{~B}$ & 1.968675 & 1.968675 & 1.968675 & 1.968675 & 1.968675 & & & & \\
\hline
\end{tabular}

\section{8-2}

\begin{tabular}{|c|c|c|c|c|c|c|c|c|c|}
\hline & 1 & 2 & 3 & 4 & 5 & & 6 & 7 & 8 \\
\hline $1 \mathrm{~B}$ & 0.000000 & & & & & $6 \mathrm{~B}$ & 0.000000 & & \\
\hline $2 \mathrm{~B}$ & 1.844313 & 0.000000 & & & & $7 \mathrm{~B}$ & 1.683147 & 0.000000 & \\
\hline $3 \mathrm{~B}$ & 1.758419 & 1.758419 & 0.000000 & & & $8 \mathrm{~B}$ & 1.979214 & 1.683147 & 0.000000 \\
\hline $4 \mathrm{~B}$ & 2.652638 & 1.840163 & 1.683147 & 0.000000 & & & & & \\
\hline $5 \mathrm{~B}$ & 1.840163 & 2.652638 & 1.683147 & 1.979214 & 0.000000 & & & & \\
\hline $6 \mathrm{~B}$ & 1.840163 & 2.652638 & 2.769066 & 2.595994 & 1.679850 & & & & \\
\hline $7 \mathrm{~B}$ & 1.758419 & 1.758419 & 2.878080 & 2.769066 & 2.769066 & & & & \\
\hline $8 \mathrm{~B}$ & 2.652638 & 1.840163 & 2.769066 & 1.679850 & 2.595994 & & & & \\
\hline
\end{tabular}

\section{8-3}

\begin{tabular}{|cccccc|ccccc|}
\hline & 1 & 2 & 3 & 4 & 5 & & 6 & 7 & 8 \\
1 B & 0.000000 & & & & & & 6.000000 & & \\
2 B & 1.614396 & 0.000000 & & & & & & \\
3 B & 2.796216 & 1.614396 & 0.000000 & & & & & & \\
3 B & 1.968675 & 0.000000 & \\
4 B & 3.228792 & 2.796216 & 1.614396 & 0.000000 & & & & & & \\
5 B & 2.796216 & 3.228792 & 2.796216 & 1.614396 & 0.000000 & & & & & \\
6 B & 1.614396 & 2.796216 & 3.228792 & 2.796216 & 1.614396 & & & & \\
7 B & 1.968675 & 1.968675 & 1.968675 & 1.968675 & 1.968675 & & & & \\
8 B & 1.968675 & 1.968675 & 1.968675 & 1.968675 & 1.968675 & & & & \\
\hline
\end{tabular}


18-4

\begin{tabular}{|c|c|c|c|c|c|c|c|c|c|}
\hline & 1 & 2 & 3 & 4 & 5 & & 6 & 7 & 8 \\
\hline $1 \mathrm{~B}$ & 0.000000 & & & & & $6 \mathrm{~B}$ & 0.000000 & & \\
\hline $2 \mathrm{~B}$ & 2.605880 & 0.000000 & & & & $7 \mathrm{~B}$ & 1.646484 & 0.000000 & \\
\hline $3 \mathrm{~B}$ & 1.739870 & 3.087166 & 0.000000 & & & $8 \mathrm{~B}$ & 2.843548 & 1.869033 & 0.000000 \\
\hline $4 \mathrm{~B}$ & 1.732149 & 4.174223 & 1.793705 & 0.000000 & & & & & \\
\hline $5 \mathrm{~B}$ & 1.632554 & 1.638456 & 2.996410 & 3.332543 & 0.000000 & & & & \\
\hline $6 \mathrm{~B}$ & 2.756899 & 4.458719 & 1.816748 & 1.697094 & 4.126289 & & & & \\
\hline $7 \mathrm{~B}$ & 2.822199 & 3.436769 & 1.815701 & 2.771077 & 3.640204 & & & & \\
\hline $8 \mathrm{~B}$ & 1.748869 & 1.640518 & 1.754248 & 2.859871 & 1.916951 & & & & \\
\hline
\end{tabular}

\section{B/H connectivities}

18-1

\begin{tabular}{|c|c|c|c|c|c|c|c|c|c|}
\hline & 1 & 2 & 3 & 4 & 5 & & 6 & 7 & 8 \\
\hline $9 \mathrm{H}$ & 2.920501 & 2.681512 & 1.220610 & 2.553447 & 3.803013 & $9 \mathrm{H}$ & 2.920501 & 2.681512 & 3.744581 \\
\hline $10 \mathrm{H}$ & 2.920501 & 2.681512 & 3.744581 & 3.803013 & 2.553447 & $10 \mathrm{H}$ & 2.920501 & 2.681512 & 1.220610 \\
\hline $11 \mathrm{H}$ & 2.821369 & 4.010347 & 3.851997 & 2.574005 & 1.216643 & $11 \mathrm{H}$ & 2.821369 & 4.010347 & 2.592253 \\
\hline $12 \mathrm{H}$ & 2.821369 & 4.010347 & 2.592253 & 1.216643 & 2.574005 & $12 \mathrm{H}$ & 2.821369 & 4.010347 & 3.851997 \\
\hline $13 \mathrm{H}$ & 2.592253 & 1.216643 & 2.821369 & 4.010347 & 4.010347 & $13 \mathrm{H}$ & 3.851997 & 2.574005 & 2.821369 \\
\hline $14 \mathrm{H}$ & 3.851997 & 2.574005 & 2.821369 & 4.010347 & 4.010347 & $14 \mathrm{H}$ & 2.592253 & 1.216643 & 2.821369 \\
\hline $15 \mathrm{H}$ & 3.744581 & 3.803013 & 2.920501 & 2.681512 & 2.681512 & $15 \mathrm{H}$ & 1.220610 & 2.553447 & 2.920501 \\
\hline $16 \mathrm{H}$ & 1.220610 & 2.553447 & 2.920501 & 2.681512 & 2.681512 & $16 \mathrm{H}$ & 3.744581 & 3.803013 & 2.920501 \\
\hline
\end{tabular}

18-2

\begin{tabular}{|ccccccc|cccc|}
\hline & & 1 & 2 & 3 & 4 & 5 & & 6 & 7 & 8 \\
$9 \mathrm{H}$ & 2.706831 & 2.706831 & 4.014337 & 3.926207 & 3.926207 & 9 & $\mathrm{H}$ & 2.621206 & 1.214848 & 2.621206 \\
$10 \mathrm{H}$ & 2.706831 & 2.706831 & 1.214848 & 2.621206 & 2.621206 & $10 \mathrm{H}$ & 3.926207 & 4.014337 & 3.926207 \\
$11 \mathrm{H}$ & 1.218333 & 2.834495 & 2.620927 & 3.824761 & 2.756750 & $11 \mathrm{H}$ & 2.756750 & 2.620927 & 3.824761 \\
$12 \mathrm{H}$ & 2.834495 & 1.218333 & 2.620927 & 2.756750 & 3.824761 & $12 \mathrm{H}$ & 3.824761 & 2.620927 & 2.756750 \\
$13 \mathrm{H}$ & 2.843274 & 3.829557 & 2.533769 & 2.924937 & 1.221706 & $13 \mathrm{H}$ & 2.602028 & 3.932268 & 3.719306 \\
$14 \mathrm{H}$ & 3.829557 & 2.843274 & 3.932268 & 2.602028 & 3.719306 & $14 \mathrm{H}$ & 2.924937 & 2.533769 & 1.221706 \\
$15 \mathrm{H}$ & 3.829557 & 2.843274 & 2.533769 & 1.221706 & 2.924937 & $15 \mathrm{H}$ & 3.719306 & 3.932268 & 2.602028 \\
$16 \mathrm{H}$ & 2.843274 & 3.829557 & 3.932268 & 3.719306 & 2.602028 & $16 \mathrm{H}$ & 1.221706 & 2.533769 & 2.924937 \\
\hline
\end{tabular}

18-3

\begin{tabular}{|c|c|c|c|c|c|c|c|c|c|}
\hline & 1 & 2 & 3 & 4 & 5 & & 6 & 7 & 8 \\
\hline $9 \mathrm{H}$ & 2.846367 & 2.846367 & 2.846367 & 2.846367 & 2.846367 & $9 \mathrm{H}$ & 2.846367 & 3.470934 & 1.217576 \\
\hline $10 \mathrm{H}$ & 2.846367 & 2.846367 & 2.846367 & 2.846367 & 2.846367 & $10 \mathrm{H}$ & 2.846367 & 1.217576 & 3.470934 \\
\hline $11 \mathrm{H}$ & 2.468090 & 1.226706 & 2.468090 & 3.907019 & 4.455498 & $11 \mathrm{H}$ & 3.907019 & 3.056348 & 3.056348 \\
\hline $12 \mathrm{H}$ & 3.907019 & 4.455498 & 3.907019 & 2.468090 & 1.226706 & $12 \mathrm{H}$ & 2.468090 & 3.056348 & 3.056348 \\
\hline $13 \mathrm{H}$ & 3.907019 & 2.468090 & 1.226706 & 2.468090 & 3.907019 & $13 \mathrm{H}$ & 4.455498 & 3.056348 & 3.056348 \\
\hline $14 \mathrm{H}$ & 2.468090 & 3.907019 & 4.455498 & 3.907019 & 2.468090 & $14 \mathrm{H}$ & 1.226706 & 3.056348 & 3.056348 \\
\hline $15 \mathrm{H}$ & 4.455498 & 3.907019 & 2.468090 & 1.226706 & 2.468090 & $15 \mathrm{H}$ & 3.907019 & 3.056348 & 3.056348 \\
\hline $16 \mathrm{H}$ & 1.226706 & 2.468090 & 3.907019 & 4.455498 & 3.907019 & $16 \mathrm{H}$ & 2.468090 & 3.056348 & 3.0563483 \\
\hline
\end{tabular}


18-4

\begin{tabular}{|ccccccc|cccc|}
\hline & & 1 & 2 & 3 & 4 & 5 & & 6 & 7 & 8 \\
9 & $\mathrm{H}$ & 2.510835 & 2.579820 & 4.067082 & 4.016772 & 1.230450 & $9 \mathrm{H}$ & 4.898097 & 4.500065 & 2.940237 \\
10 & $\mathrm{H}$ & 3.884744 & 5.579672 & 2.726758 & 2.551013 & 5.322434 & $10 \mathrm{H}$ & 1.226459 & 2.528153 & 3.976779 \\
11 & $\mathrm{H}$ & 3.935378 & 3.952375 & 2.740360 & 3.931655 & 4.550369 & $11 \mathrm{H}$ & 2.609691 & 1.219745 & 2.655114 \\
12 & $\mathrm{H}$ & 2.597310 & 3.513490 & 1.213929 & 2.659129 & 3.694968 & $12 \mathrm{H}$ & 2.730757 & 2.725912 & 2.588862 \\
13 & $\mathrm{H}$ & 3.814190 & 1.214950 & 4.129538 & 5.363615 & 2.718369 & $13 \mathrm{H}$ & 5.519695 & 4.311024 & 2.702300 \\
14 & $\mathrm{H}$ & 1.371277 & 3.762802 & 2.455334 & 1.304963 & 2.567872 & $14 \mathrm{H}$ & 2.491188 & 3.084220 & 2.671256 \\
15 & $\mathrm{H}$ & 2.607691 & 2.708884 & 2.428995 & 3.162966 & 2.865229 & $15 \mathrm{H}$ & 2.571172 & 1.323008 & 1.374684 \\
16 & $\mathrm{H}$ & 2.584583 & 5.137525 & 2.767233 & 1.217508 & 4.140915 & $16 \mathrm{H}$ & 2.646856 & 3.936598 & 3.983009 \\
\hline
\end{tabular}


Table S2. B/B and B/H Connectivities of $\mathrm{B}_{8} \mathrm{H}_{8}{ }^{4-}$

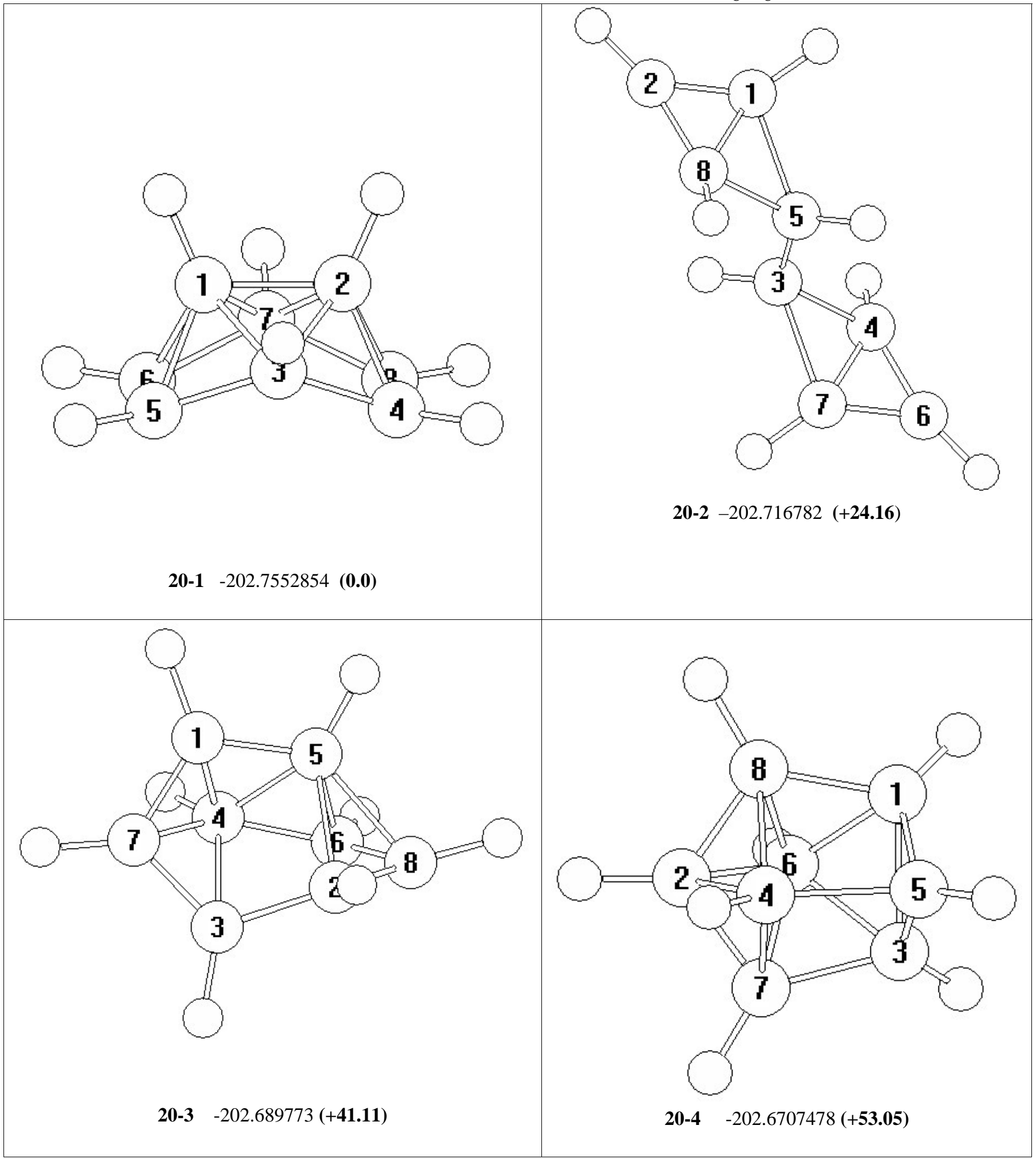




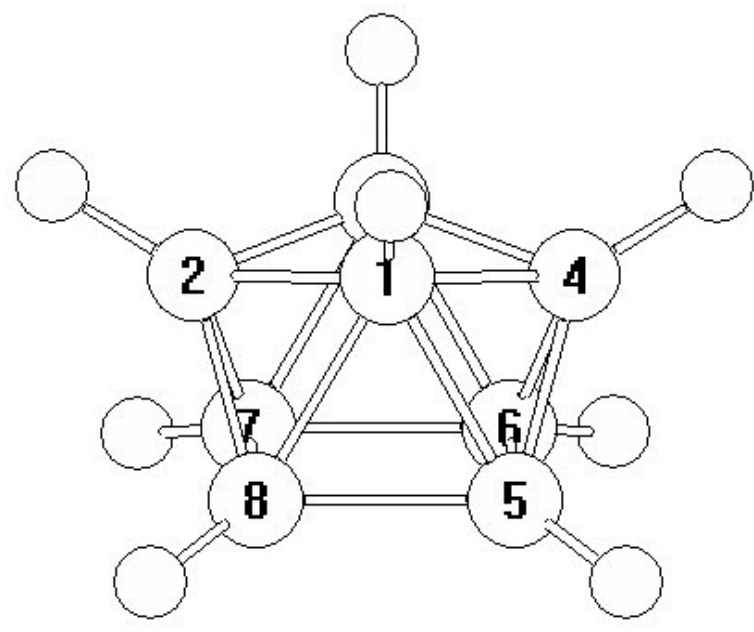

$\mathbf{2 0 - 5} \quad-202.6656807(+\mathbf{5 6 . 2 3})$

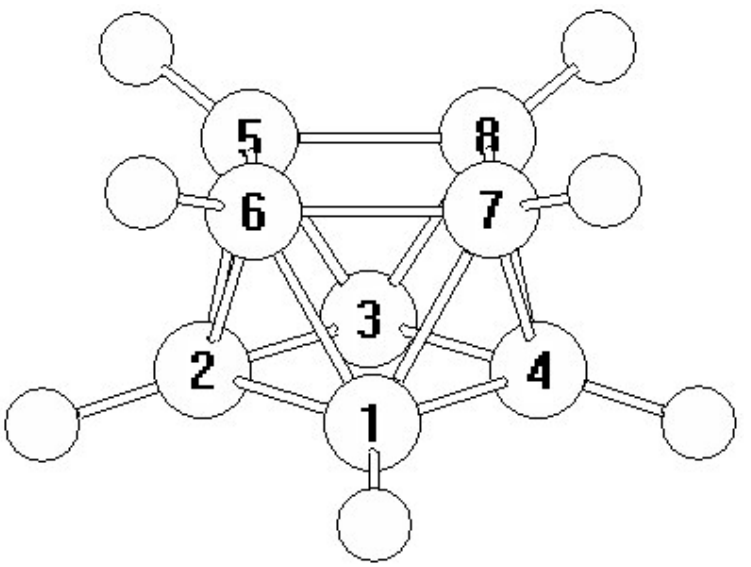

20-6 -202.6447785 (+69.34)

\section{B/B connectivities}

20-1

\begin{tabular}{|c|c|c|c|c|c|c|c|c|c|}
\hline & 1 & 2 & 3 & 4 & 5 & & 6 & 7 & 8 \\
\hline $1 \mathrm{~B}$ & 0.000000 & & & & & $6 \mathrm{~B}$ & 0.000000 & & \\
\hline $2 \mathrm{~B}$ & 1.778649 & 0.000000 & & & & $7 \mathrm{~B}$ & 1.792300 & 0.000000 & \\
\hline $3 \mathrm{~B}$ & 1.868229 & 1.868229 & 0.000000 & & & $8 \mathrm{~B}$ & 3.099475 & 1.792300 & 0.000000 \\
\hline $4 \mathrm{~B}$ & 2.953808 & 1.792234 & 1.792300 & 0.000000 & & & & & \\
\hline $5 \mathrm{~B}$ & 1.792234 & 2.953808 & 1.792300 & 3.099475 & 0.000000 & & & & \\
\hline $6 \mathrm{~B}$ & 1.792234 & 2.953808 & 2.818700 & 3.508742 & 1.644544 & & & & \\
\hline $7 \mathrm{~B}$ & 1.868229 & 1.868229 & 2.877838 & 2.818700 & 2.818700 & & & & \\
\hline $8 \mathrm{~B}$ & 2.953808 & 1.792234 & 2.818700 & 1.644544 & 3.508742 & & & & \\
\hline
\end{tabular}

\section{0-2}

\begin{tabular}{|c|c|c|c|c|c|c|c|c|c|}
\hline & 1 & 2 & 3 & 4 & 5 & & 6 & 7 & 8 \\
\hline $1 \mathrm{~B}$ & 0.000000 & & & & &. $.6 \mathrm{~B}$ & 0.000000 & & \\
\hline $2 \mathrm{~B}$ & 1.609263 & 0.000000 & & & & $7 \mathrm{~B}$ & 1.609302 & 0.000000 & \\
\hline $3 \mathrm{~B}$ & 3.204729 & 4.054724 & 0.000000 & & & $8 \mathrm{~B}$ & 5.864256 & 4.746012 & 0.000000 \\
\hline $4 \mathrm{~B}$ & 4.752180 & 5.866031 & 1.957194 & 0.000000 & & & & & \\
\hline $5 \mathrm{~B}$ & 1.956359 & 3.028292 & 1.652922 & 3.206011 & 0.000000 & & & & \\
\hline $6 \mathrm{~B}$ & 5.867120 & 6.965428 & 3.029600 & 1.609184 & 4.057040 & & & & \\
\hline $7 \mathrm{~B}$ & 5.045743 & 5.862940 & 1.956826 & 1.704830 & 3.203888 & & & & \\
\hline $8 \mathrm{~B}$ & 1.705104 & 1.609346 & 3.202934 & 5.045998 & 1.956117 & & & & \\
\hline
\end{tabular}


20-3

\begin{tabular}{|c|c|c|c|c|c|c|c|c|c|}
\hline & 1 & 2 & 3 & 4 & 5 & & 6 & 7 & 8 \\
\hline $1 \mathrm{~B}$ & .000000 & & & & & $6 \mathrm{~B}$ & .000000 & & \\
\hline $2 \mathrm{~B}$ & 2.869315 & .000000 & & & & $7 \mathrm{~B}$ & 3.470638 & .000000 & \\
\hline $3 \mathrm{~B}$ & 2.619445 & 1.787114 & .000000 & & & $8 \mathrm{~B}$ & 1.673382 & 3.916163 & .000000 \\
\hline $4 \mathrm{~B}$ & 1.875512 & 2.808871 & 2.178168 & .000000 & & & & & \\
\hline $5 \mathrm{~B}$ & 1.736604 & 2.178168 & 2.808871 & 1.865427 & .000000 & & & & \\
\hline $6 \mathrm{~B}$ & 3.093208 & 2.619445 & 2.869315 & 1.736604 & 1.875512 & & & & \\
\hline $7 \mathrm{~B}$ & 1.673382 & 2.910720 & 1.663215 & 1.986741 & 2.857410 & & & & \\
\hline $8 \mathrm{~B}$ & 3.470638 & 1.663215 & 2.910720 & 2.857410 & 1.986741 & & & & \\
\hline
\end{tabular}

\section{0-4}

\begin{tabular}{|c|c|c|c|c|c|c|c|c|c|}
\hline & 1 & 2 & 3 & 4 & 5 & & 6 & 7 & 8 \\
\hline $1 \mathrm{~B}$ & 0.000000 & & & & & $6 \mathrm{~B}$ & 0.000000 & & \\
\hline $2 \mathrm{~B}$ & 2.880980 & 0.000000 & & & & $7 \mathrm{~B}$ & 1.847304 & 0.000000 & \\
\hline $3 \mathrm{~B}$ & 1.972280 & 2.881541 & 0.000000 & & & $8 \mathrm{~B}$ & 1.847582 & 2.744680 & 0.000000 \\
\hline $4 \mathrm{~B}$ & 2.598820 & 1.807139 & 2.598209 & 0.000000 & & & & & \\
\hline $5 \mathrm{~B}$ & 1.730275 & 3.151564 & 1.730106 & 1.931725 & 0.000000 & & & & \\
\hline $6 \mathrm{~B}$ & 1.879531 & 1.746182 & 1.879512 & 2.614611 & 2.763468 & & & & \\
\hline $7 \mathrm{~B}$ & 2.939241 & 1.682238 & 1.796489 & 1.972883 & 2.629409 & & & & \\
\hline $8 \mathrm{~B}$ & 1.795917 & 1.681929 & 2.939406 & 1.974708 & 2.629540 & & & & \\
\hline
\end{tabular}

\section{0-5}

\begin{tabular}{|c|c|c|c|c|c|c|c|c|c|}
\hline & 1 & 2 & 3 & 4 & 5 & & 6 & 7 & 8 \\
\hline $1 \mathrm{~B}$ & 0.000000 & & & & & $6 \mathrm{~B}$ & 0.000000 & & \\
\hline $2 \mathrm{~B}$ & 1.728564 & 0.000000 & & & & $7 \mathrm{~B}$ & 1.951094 & 0.000000 & \\
\hline $3 \mathrm{~B}$ & 1.852975 & 1.728439 & 0.000000 & & & $8 \mathrm{~B}$ & 2.617452 & 1.745311 & 0.000000 \\
\hline $4 \mathrm{~B}$ & 1.728439 & 2.863711 & 1.728564 & 0.000000 & & & & & \\
\hline $5 \mathrm{~B}$ & 2.016016 & 2.959030 & 2.700833 & 1.778046 & 0.000000 & & & & \\
\hline $6 \mathrm{~B}$ & 2.700544 & 2.957048 & 2.013725 & 1.778711 & 1.745311 & & & & \\
\hline $7 \mathrm{~B}$ & 2.700833 & 1.778046 & 2.016016 & 2.959030 & 2.618147 & & & & \\
\hline $8 \mathrm{~B}$ & 2.013725 & 1.778711 & 2.700544 & 2.957048 & 1.951094 & & & & \\
\hline
\end{tabular}

\section{0-6}

\begin{tabular}{|c|c|c|c|c|c|c|c|c|c|}
\hline & 1 & 2 & 3 & 4 & 5 & & 6 & 7 & 8 \\
\hline $1 \mathrm{~B}$ & 0.000000 & & & & & $6 \mathrm{~B}$ & 0.000000 & & \\
\hline $2 \mathrm{~B}$ & 1.802179 & 0.000000 & & & & $7 \mathrm{~B}$ & 1.802180 & 0.000000 & \\
\hline $3 \mathrm{~B}$ & 2.548666 & 1.802179 & 0.000000 & & & $8 \mathrm{~B}$ & 2.548667 & 1.802180 & 0.000000 \\
\hline $4 \mathrm{~B}$ & 1.802179 & 2.548666 & 1.802179 & 0.000000 & & & & & \\
\hline $5 \mathrm{~B}$ & 2.827794 & 1.844794 & 1.844794 & 2.827794 & 0.000000 & & & & \\
\hline $6 \mathrm{~B}$ & 1.844794 & 1.844794 & 2.827794 & 2.827794 & 1.802180 & & & & \\
\hline $7 \mathrm{~B}$ & 1.844794 & 2.827794 & 2.827794 & 1.844794 & 2.548667 & & & & \\
\hline $8 \mathrm{~B}$ & 2.827794 & 2.827794 & 1.844794 & 1.844794 & 1.802180 & & & & \\
\hline
\end{tabular}




\section{B/H connectivities}

20-1
\begin{tabular}{|cccccc|cc|ccc|}
\hline & & 1 & 2 & 3 & 4 & 5 & & 6 & 7 & 8 \\
9 & $\mathrm{H}$ & 2.716746 & 2.716746 & 4.051574 & 3.940611 & 3.940611 & $9 \mathrm{H}$ & 2.664728 & 1.291305 & 2.664728 \\
10 & $\mathrm{H}$ & 2.716746 & 2.716746 & 1.291305 & 2.664728 & 2.664728 & $10 \mathrm{H}$ & 3.940611 & 4.051574 & 3.940611 \\
11 & $\mathrm{H}$ & 1.278097 & 2.566940 & 2.807645 & 4.027432 & 2.753996 & $11 \mathrm{H}$ & 2.753996 & 2.807645 & 4.027432 \\
12 & $\mathrm{H}$ & 2.566940 & 1.278097 & 2.807645 & 2.753996 & 4.027432 & $12 \mathrm{H}$ & 4.027432 & 2.807645 & 2.753996 \\
13 & $\mathrm{H}$ & 2.745384 & 4.093612 & 2.679948 & 4.211378 & 1.291517 & $13 \mathrm{H}$ & 2.623153 & 4.037865 & 4.790472 \\
14 & $\mathrm{H}$ & 4.093612 & 2.745384 & 4.037865 & 2.623153 & 4.790472 & $14 \mathrm{H}$ & 4.211378 & 2.679948 & 1.291517 \\
15 & $\mathrm{H}$ & 4.093612 & 2.745384 & 2.679948 & 1.291517 & 4.211378 & $15 \mathrm{H}$ & 4.790472 & 4.037865 & 2.623153 \\
16 & $\mathrm{H}$ & 2.745384 & 4.093612 & 4.037865 & 4.790472 & 2.623153 & $16 \mathrm{H}$ & 1.291517 & 2.679948 & 4.211378 \\
\hline
\end{tabular}

20-2

\begin{tabular}{|c|c|c|c|c|c|c|c|c|c|}
\hline & 1 & 2 & 3 & 4 & 5 & & 6 & 7 & 8 \\
\hline $9 \mathrm{H}$ & 2.639559 & 3.863182 & 2.528870 & 3.519772 & 1.259393 & $9 \mathrm{H}$ & 3.994952 & 3.517382 & 2.640027 \\
\hline $10 \mathrm{H}$ & 7.081289 & 8.213601 & 4.287512 & 2.721679 & 5.271831 & $10 \mathrm{H}$ & 1.259788 & 2.721886 & 7.078202 \\
\hline $11 \mathrm{H}$ & 5.548822 & 6.052038 & 2.570740 & 2.838619 & 3.794849 & $11 \mathrm{H}$ & 2.690078 & 1.256104 & 4.927051 \\
\hline $12 \mathrm{H}$ & 3.518641 & 3.992330 & 1.259261 & 2.639634 & 2.528943 & $12 \mathrm{H}$ & 3.863305 & 2.640049 & 3.516581 \\
\hline $13 \mathrm{H}$ & 2.721874 & 1.259875 & 5.269516 & 7.080188 & 4.286285 & $13 \mathrm{H}$ & 8.213547 & 7.076752 & 2.721640 \\
\hline $14 \mathrm{H}$ & 1.256395 & 2.690160 & 3.799041 & 4.938893 & 2.571598 & $14 \mathrm{H}$ & 6.061340 & 5.553104 & 2.838868 \\
\hline $15 \mathrm{H}$ & 2.838581 & 2.690031 & 3.796038 & 5.550989 & 2.571299 & $15 \mathrm{H}$ & 6.055548 & 4.929506 & 1.256288 \\
\hline $16 \mathrm{H}$ & 4.936344 & 6.057905 & 2.570928 & 1.256166 & 3.798106 & $16 \mathrm{H}$ & 2.690280 & 2.838851 & 5.551303 \\
\hline
\end{tabular}

20-3

\begin{tabular}{|ccccccc|ccccc|}
\hline & & 1 & 2 & 3 & 4 & 5 & & & 6 & 7 & 8 \\
9 & $\mathrm{H}$ & 2.651086 & 4.179939 & 2.690867 & 2.822184 & 4.034064 & 9 & $\mathrm{H}$ & 4.470652 & 1.309398 & 5.145388 \\
10 & $\mathrm{H}$ & 4.470652 & 2.690867 & 4.179939 & 4.034064 & 2.822184 & 10 & $\mathrm{H}$ & 2.651086 & 5.145388 & 1.309398 \\
11 & $\mathrm{H}$ & 1.306314 & 4.096174 & 3.900185 & 2.821035 & 2.578231 & 11 & $\mathrm{H}$ & 4.026804 & 2.683829 & 4.524715 \\
12 & $\mathrm{H}$ & 4.026804 & 3.900185 & 4.096174 & 2.578231 & 2.821035 & 12 & $\mathrm{H}$ & 1.306314 & 4.524715 & 2.683829 \\
13 & $\mathrm{H}$ & 3.530228 & 1.306760 & 2.684675 & 3.976163 & 3.063893 & 13 & $\mathrm{H}$ & 3.869989 & 3.622719 & 2.643481 \\
14 & $\mathrm{H}$ & 3.869989 & 2.684675 & 1.306760 & 3.063893 & 3.976163 & 14 & $\mathrm{H}$ & 3.530228 & 2.643481 & 3.622719 \\
15 & $\mathrm{H}$ & 2.521426 & 3.244029 & 4.079423 & 2.879510 & 1.272630 & 15 & $\mathrm{H}$ & 2.620212 & 3.966689 & 2.678944 \\
16 & $\mathrm{H}$ & 2.620212 & 4.079423 & 3.244029 & 1.272630 & 2.879510 & 16 & $\mathrm{H}$ & 2.521426 & 2.678944 & 3.966689 \\
\hline
\end{tabular}

\section{0-4}

\begin{tabular}{|ccccccc|cccc|}
\hline & & 1 & 2 & 3 & 4 & 5 & & 6 & 7 & 8 \\
9 & $\mathrm{H}$ & 2.794470 & 2.494907 & 4.189325 & 3.041280 & 3.815904 & $9 \mathrm{H}$ & 2.840216 & 3.900294 & 1.287955 \\
10 & $\mathrm{H}$ & 1.300914 & 3.966108 & 2.825229 & 3.895067 & 2.817395 & $10 \mathrm{H}$ & 2.645177 & 4.046810 & 2.760774 \\
11 & $\mathrm{H}$ & 2.793657 & 2.642516 & 2.793757 & 3.857372 & 3.940198 & $11 \mathrm{H}$ & 1.272281 & 2.804313 & 2.804265 \\
12 & $\mathrm{H}$ & 3.815082 & 2.596730 & 3.814639 & 1.296942 & 2.967607 & $12 \mathrm{H}$ & 3.824460 & 2.929564 & 2.931114 \\
13 & $\mathrm{H}$ & 2.825135 & 3.966512 & 1.300927 & 3.894416 & 2.817436 & $13 \mathrm{H}$ & 2.644773 & 2.761443 & 4.046651 \\
14 & $\mathrm{H}$ & 4.188956 & 2.495219 & 2.794778 & 3.039708 & 3.816008 & $14 \mathrm{H}$ & 2.839252 & 1.287978 & 3.900190 \\
15 & $\mathrm{H}$ & 2.752632 & 4.368405 & 2.753054 & 2.886100 & 1.285497 & $15 \mathrm{H}$ & 4.032050 & 3.760901 & 3.760341 \\
16 & $\mathrm{H}$ & 4.078442 & 1.262078 & 4.078958 & 2.779067 & 4.372648 & $16 \mathrm{H}$ & 2.798520 & 2.622542 & 2.622419 \\
\hline
\end{tabular}


20-5

\begin{tabular}{|ccccccc|ccccc|}
\hline & & 1 & 2 & 3 & 4 & 5 & & & 6 & 7 & 8 \\
9 & $\mathrm{H}$ & 2.929273 & 2.621872 & 1.283525 & 2.621808 & 3.924573 & 9 & $\mathrm{H}$ & 2.977299 & 2.980015 & 3.924695 \\
10 & $\mathrm{H}$ & 2.897505 & 2.594710 & 3.887141 & 4.116586 & 2.935899 & 10 & $\mathrm{H}$ & 3.864635 & 2.822865 & 1.285532 \\
11 & $\mathrm{H}$ & 2.901079 & 4.118906 & 3.887791 & 2.595048 & 1.285053 & 11 & $\mathrm{H}$ & 2.822951 & 3.864148 & 2.934945 \\
12 & $\mathrm{H}$ & 2.692504 & 3.986408 & 2.691618 & 1.268093 & 2.791402 & 12 & $\mathrm{H}$ & 2.791514 & 4.186944 & 4.185330 \\
13 & $\mathrm{H}$ & 2.691618 & 1.268093 & 2.692504 & 3.986408 & 4.186944 & 13 & $\mathrm{H}$ & 4.185330 & 2.791402 & 2.791514 \\
14 & $\mathrm{H}$ & 3.887791 & 2.595048 & 2.901079 & 4.118906 & 3.864148 & 14 & $\mathrm{H}$ & 2.934945 & 1.285053 & 2.822951 \\
15 & $\mathrm{H}$ & 3.887141 & 4.116586 & 2.897505 & 2.594710 & 2.822865 & 15 & $\mathrm{H}$ & 1.285532 & 2.935899 & 3.864635 \\
16 & $\mathrm{H}$ & 1.283525 & 2.621808 & 2.929273 & 2.621872 & 2.980015 & 16 & $\mathrm{H}$ & 3.924695 & 3.924573 & 2.977299 \\
\hline
\end{tabular}

\section{0-6}

\begin{tabular}{|c|c|c|c|c|c|c|c|c|c|}
\hline & 1 & 2 & 3 & 4 & 5 & & 6 & 7 & 8 \\
\hline $9 \mathrm{H}$ & 2.821462 & 1.280276 & 2.821462 & 3.779178 & 2.699714 & $9 \mathrm{H}$ & 2.699714 & 4.028457 & 4.028457 \\
\hline $10 \mathrm{H}$ & 1.280276 & 2.821462 & 3.779178 & 2.821462 & 4.028457 & $10 \mathrm{H}$ & 2.699714 & 2.699714 & 4.028457 \\
\hline $11 \mathrm{H}$ & 3.779178 & 2.821462 & 1.280276 & 2.821462 & 2.699714 & $11 \mathrm{H}$ & 4.028457 & 4.028457 & 2.699714 \\
\hline $12 \mathrm{H}$ & 2.821462 & 3.779178 & 2.821462 & 1.280276 & 4.028457 & $12 \mathrm{H}$ & 4.028457 & 2.699714 & 2.699714 \\
\hline $13 \mathrm{H}$ & 2.699714 & 2.699714 & 4.028456 & 4.028456 & 2.821462 & $13 \mathrm{H}$ & 1.280275 & 2.821462 & 3.779178 \\
\hline $14 \mathrm{H}$ & 4.028456 & 4.028456 & 2.699714 & 2.699714 & 2.821462 & $14 \mathrm{H}$ & 3.779178 & 2.821462 & 1.280275 \\
\hline $15 \mathrm{H}$ & 4.028456 & 2.699714 & 2.699714 & 4.028456 & 1.280275 & $15 \mathrm{H}$ & 2.821462 & 3.779178 & 2.821462 \\
\hline $16 \mathrm{H}$ & 2.699714 & 4.028456 & 4.028456 & 2.699714 & 3.779178 & $16 \mathrm{H}$ & 2.821462 & 1.280275 & 2.821462 \\
\hline
\end{tabular}


Table S3. B/B and B/H Connectivities of $\mathrm{B}_{11} \mathrm{H}_{11^{2-}}$

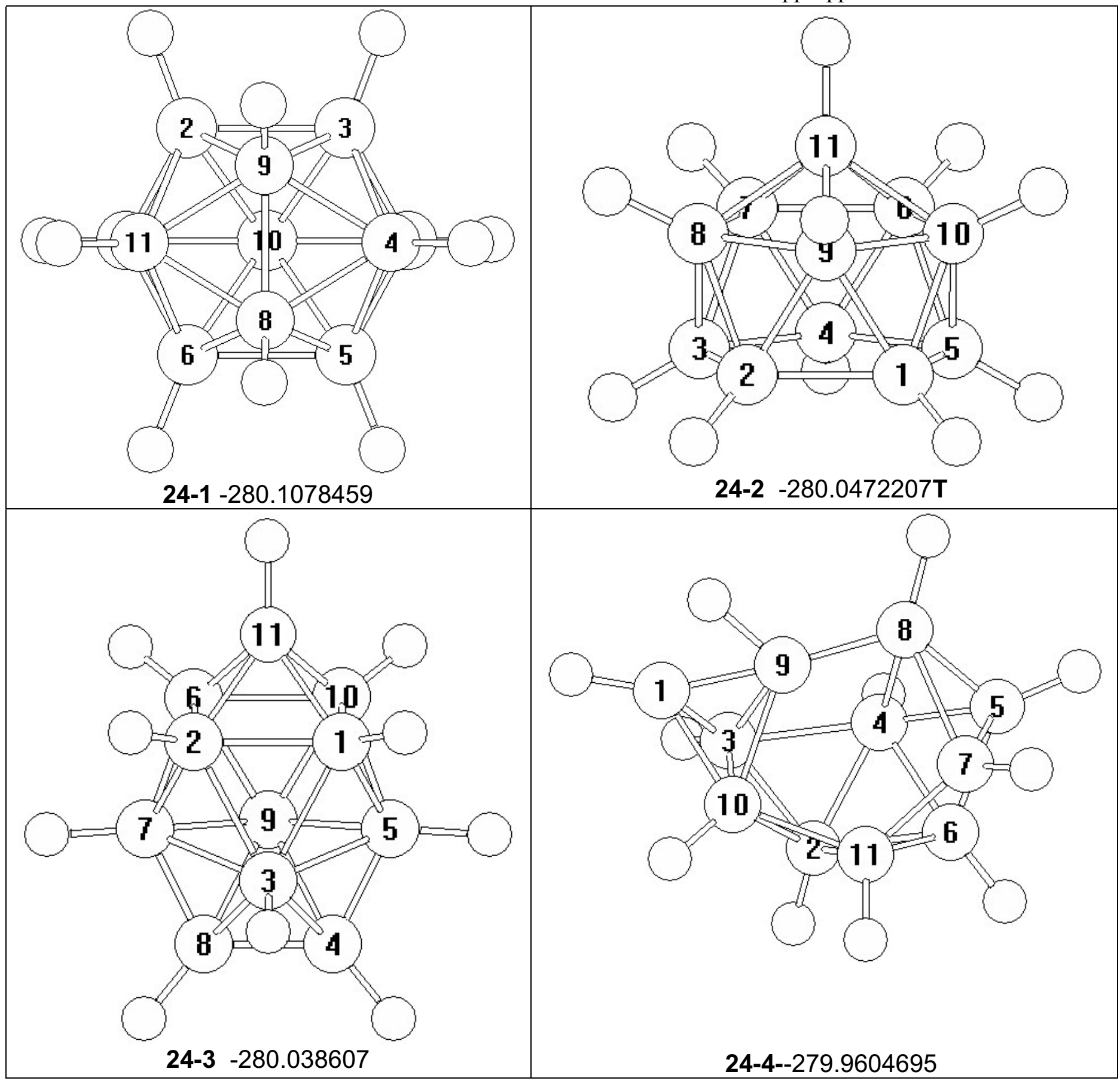




\section{B/B connectivities}

\begin{tabular}{|c|c|c|c|c|c|c|c|c|c|c|}
\hline & 1 & 2 & 3 & 4 & 5 & 6 & 7 & 8 & 9 & 10 \\
\hline $1 \mathrm{~B}$ & 0.000000 & & & & & & & & & \\
\hline $2 \mathrm{~B}$ & 1.669928 & 0.000000 & & & & & & & & \\
\hline $3 \mathrm{~B}$ & 3.004032 & 1.870588 & 0.000000 & & & & & & & \\
\hline $4 \mathrm{~B}$ & 3.604686 & 2.962128 & 1.794234 & 0.000000 & & & & & & \\
\hline $5 \mathrm{~B}$ & 3.004032 & 3.261230 & 2.671427 & 1.794234 & 0.000000 & & & & & \\
\hline $6 \mathrm{~B}$ & 1.669928 & 2.671427 & 3.261230 & 2.962128 & 1.870588 & 0.000000 & & & & \\
\hline $7 \mathrm{~B}$ & 3.333471 & 3.004032 & 1.669928 & 1.759159 & 1.669928 & 3.004032 & 0.000000 & & & \\
\hline $8 \mathrm{~B}$ & 2.867896 & 2.842386 & 2.842386 & 1.789010 & 1.785324 & 1.785324 & 2.867896 & 0.000000 & & \\
\hline $9 \mathrm{~B}$ & 2.867896 & 1.785324 & 1.785324 & 1.789010 & 2.842386 & 2.842386 & 2.867896 & 1.831146 & 0.000000 & \\
\hline $10 \mathrm{~B}$ & 1.745071 & 2.025875 & 2.025875 & 2.709721 & 2.025875 & 2.025875 & 1.745071 & 2.816686 & 2.816686 & 0.000000 \\
\hline $11 \mathrm{~B}$ & 1.759159 & 1.794234 & 2.962128 & 2.969614 & 2.962128 & 1.794234 & 3.604686 & 1.789010 & 1.789010 & 2.709721 \\
\hline
\end{tabular}

\section{4-2}

\begin{tabular}{|c|c|c|c|c|c|c|c|c|c|c|}
\hline & 1 & 2 & 3 & 4 & 5 & 6 & 7 & 8 & 9 & 10 \\
\hline $1 \mathrm{~B}$ & 0.000000 & & & & & & & & & \\
\hline $2 \mathrm{~B}$ & 1.784116 & 0.000000 & & & & & & & & \\
\hline $3 \mathrm{~B}$ & 2.886760 & 1.784116 & 0.000000 & & & & & & & \\
\hline $4 \mathrm{~B}$ & 2.886760 & 2.886760 & 1.784116 & 0.000000 & & & & & & \\
\hline $5 \mathrm{~B}$ & 1.784116 & 2.886760 & 2.886760 & 1.784116 & 0.000000 & & & & & \\
\hline $6 \mathrm{~B}$ & 2.885618 & 3.396693 & 2.885618 & 1.769666 & 1.769666 & 0.000000 & & & & \\
\hline $7 \mathrm{~B}$ & 3.396693 & 2.885618 & 1.769666 & 1.769666 & 2.885618 & 1.799621 & 0.000000 & & & \\
\hline $8 \mathrm{~B}$ & 2.885618 & 1.769666 & 1.769666 & 2.885618 & 3.396693 & 2.911848 & 1.799621 & 0.000000 & & \\
\hline $9 \mathrm{~B}$ & 1.769666 & 1.769666 & 2.885618 & 3.396693 & 2.885618 & 2.911848 & 2.911848 & 1.799621 & 0.000000 & \\
\hline $10 \mathrm{~B}$ & 1.769666 & 2.885618 & 3.396693 & 2.885618 & 1.769666 & 1.799621 & 2.911848 & 2.911848 & 1.799621 & 0.000000 \\
\hline $11 \mathrm{~B}$ & 2.867037 & 2.867037 & 2.867037 & 2.867037 & 2.867037 & 1.793473 & 1.793473 & 1.793473 & 1.793473 & 1.793473 \\
\hline
\end{tabular}

\section{4-3}

\begin{tabular}{|c|c|c|c|c|c|c|c|c|c|c|}
\hline & 1 & 2 & 3 & 4 & 5 & 6 & 7 & 8 & 9 & 10 \\
\hline $1 \mathrm{~B}$ & 0.000000 & & & & & & & & & \\
\hline $2 \mathrm{~B}$ & 1.818062 & 0.000000 & & & & & & & & \\
\hline $3 \mathrm{~B}$ & 1.939777 & 1.939158 & 0.000000 & & & & & & & \\
\hline $4 \mathrm{~B}$ & 3.038581 & 3.478910 & 1.901758 & 0.000000 & & & & & & \\
\hline $5 \mathrm{~B}$ & 1.777761 & 2.939416 & 1.965913 & 1.654271 & 0.000000 & & & & & \\
\hline $6 \mathrm{~B}$ & 2.569996 & 1.816256 & 2.869753 & 3.479211 & 2.940008 & 0.000000 & & & & \\
\hline $7 \mathrm{~B}$ & 2.940008 & 1.778310 & 1.965979 & 2.738059 & 3.014473 & 1.777761 & 0.000000 & & & \\
\hline $8 \mathrm{~B}$ & 3.479211 & 3.038537 & 1.901612 & 1.579166 & 2.738059 & 3.038581 & 1.654271 & 0.000000 & & \\
\hline $9 \mathrm{~B}$ & 2.869753 & 2.869879 & 2.463479 & 1.901612 & 1.965979 & 1.939777 & 1.965913 & 1.901758 & 0.000000 & \\
\hline $10 \mathrm{~B}$ & 1.816256 & 2.569706 & 2.869879 & 3.038537 & 1.778310 & 1.818062 & 2.939416 & 3.478910 & 1.939158 & 0.000000 \\
\hline $11 \mathrm{~B}$ & 1.691916 & 1.691897 & 3.043480 & 4.075132 & 2.925553 & 1.691916 & 2.925553 & 4.075132 & 3.043480 & 1.691897 \\
\hline
\end{tabular}




\section{4-4}

\begin{tabular}{|c|c|c|c|c|c|c|c|c|c|c|}
\hline & 1 & 2 & 3 & 4 & 5 & 6 & 7 & 8 & 9 & 10 \\
\hline $1 \mathrm{~B}$ & 0.000000 & & & & & & & & & \\
\hline $2 \mathrm{~B}$ & 2.983093 & 0.000000 & & & & & & & & \\
\hline $3 \mathrm{~B}$ & 1.715645 & 1.757276 & 0.000000 & & & & & & & \\
\hline $4 \mathrm{~B}$ & 3.241079 & 1.881412 & 1.959519 & 0.000000 & & & & & & \\
\hline $5 \mathrm{~B}$ & 4.332460 & 2.970537 & 3.506452 & 1.765219 & 0.000000 & & & & & \\
\hline $6 \mathrm{~B}$ & 4.176330 & 1.780640 & 3.108231 & 1.856214 & $1.707480 \mathrm{~B}$ & 0.000000 & & & & \\
\hline $7 \mathrm{~B}$ & 4.046617 & 2.875675 & 3.691946 & 2.681752 & $1.699129 \mathrm{~B}$ & 1.804143 & 0.000000 & & & \\
\hline $8 \mathrm{~B}$ & 3.200776 & 3.176305 & 2.954273 & 1.983041 & $1.649357 \mathrm{~B}$ & 2.740424 & 2.046857 & 0.000000 & & \\
\hline $9 \mathrm{~B}$ & 1.581506 & 2.655216 & 1.898645 & 2.269520 & $2.904162 \mathrm{~B}$ & 3.162861 & 2.719313 & 1.637607 & 0.000000 & \\
\hline $10 \mathrm{~B}$ & 1.731449 & 1.791089 & 1.816997 & 2.860749 & $3.713877 \mathrm{~B}$ & 2.995128 & 3.031428 & 3.138043 & 1.889152 & 0.000000 \\
\hline $11 \mathrm{~B}$ & 3.374453 & 1.797080 & 2.974256 & 2.778339 & $2.828063 \mathrm{~B}$ & 1.786359 & 1.714025 & 2.968000 & 2.666994 & 1.822519 \\
\hline
\end{tabular}

\section{B/H connectivities}

\section{4-1}

\begin{tabular}{|cccccccccccccc}
\hline & & $\mathrm{B} 1$ & $\mathrm{~B} 2$ & $\mathrm{~B} 3$ & $\mathrm{~B} 4$ & $\mathrm{~B} 5$ & $\mathrm{~B} 6$ & $\mathrm{~B} 7$ & $\mathrm{~B} 8$ & B9 & B10 & B11 \\
1 & $\mathrm{H}$ & 2.399404 & 2.910833 & 2.910833 & 3.779608 & 2.910833 & 2.910833 & 2.399404 & 3.979525 & 3.979525 & 1.209041 & 3.779608 \\
2 & $\mathrm{H}$ & 1.207537 & 2.578385 & 4.067339 & 4.805748 & 4.067339 & 2.578385 & 4.369417 & 3.997948 & 3.997948 & 2.651828 & 2.717779 \\
3 & $\mathrm{H}$ & 4.369417 & 4.067339 & 2.578385 & 2.717779 & 2.578385 & 4.067339 & 1.207537 & 3.997948 & 3.997948 & 2.651828 & 4.805748 \\
4 & $\mathrm{H}$ & 4.802869 & 4.053614 & 2.705756 & 1.209808 & 2.705756 & 4.053614 & 2.613792 & 2.625309 & 2.625309 & 3.875831 & 3.990878 \\
5 & $\mathrm{H}$ & 2.613792 & 2.705756 & 4.053614 & 3.990878 & 4.053614 & 2.705756 & 4.802869 & 2.625309 & 2.625309 & 3.875831 & 1.209808 \\
6 & $\mathrm{H}$ & 2.528436 & 3.818100 & 4.439805 & 3.958366 & 2.568930 & 1.210547 & 3.942330 & 2.618696 & 3.980652 & 3.001155 & 2.741955 \\
7 & $\mathrm{H}$ & 3.942330 & 4.439805 & 3.818100 & 2.741955 & 1.210547 & 2.568930 & 2.528436 & 2.618696 & 3.980652 & 3.001155 & 3.958366 \\
8 & $\mathrm{H}$ & 3.942330 & 2.568930 & 1.210547 & 2.741955 & 3.818100 & 4.439805 & 2.528436 & 3.980652 & 2.618696 & 3.001155 & 3.958366 \\
9 & $\mathrm{H}$ & 2.528436 & 1.210547 & 2.568930 & 3.958366 & 4.439805 & 3.818100 & 3.942330 & 3.980652 & 2.618696 & 3.001155 & 2.741955 \\
10 & $\mathrm{H}$ & 3.895660 & 2.624338 & 2.624338 & 2.597976 & 3.951861 & 3.951861 & 3.895660 & 2.728639 & 1.208884 & 3.986269 & 2.597976 \\
11 & $\mathrm{H}$ & 3.895660 & 3.951861 & 3.951861 & 2.597976 & 2.624338 & 2.624338 & 3.895660 & 1.208884 & 2.728639 & 3.986269 & 2.597976 \\
\hline
\end{tabular}

\section{4-2T}

\begin{tabular}{|ccccccccccccc|}
\hline & & B1 & B2 & B3 & B4 & B5 & B6 & B7 & B8 & B9 & B10 & B11 \\
1 & $H$ & 2.625380 & 2.625380 & 3.968609 & 4.606944 & 3.968609 & 3.990082 & 3.990082 & 2.643207 & 1.210328 & 2.643207 & 2.638428 \\
2 & $\mathrm{H}$ & 3.944079 & 3.944079 & 3.944079 & 3.944079 & 3.944079 & 2.633094 & 2.633094 & 2.633094 & 2.633094 & 2.633094 & 1.207986 \\
3 & $\mathrm{H}$ & 3.968609 & 4.606944 & 3.968609 & 2.625380 & 2.625380 & 1.210328 & 2.643207 & 3.990082 & 3.990082 & 2.643207 & 2.638428 \\
4 & $\mathrm{H}$ & 3.934737 & 3.934737 & 2.611663 & 1.211514 & 2.611663 & 2.658147 & 2.658147 & 3.975222 & 4.604555 & 3.975222 & 3.986802 \\
5 & $\mathrm{H}$ & 2.611663 & 3.934737 & 3.934737 & 2.611663 & 1.211514 & 2.658147 & 3.975222 & 4.604555 & 3.975222 & 2.658147 & 3.986802 \\
6 & $\mathrm{H}$ & 1.211514 & 2.611663 & 3.934737 & 3.934737 & 2.611663 & 3.975222 & 4.604555 & 3.975222 & 2.658147 & 2.658147 & 3.986802 \\
7 & $\mathrm{H}$ & 2.611663 & 1.211514 & 2.611663 & 3.934737 & 3.934737 & 4.604555 & 3.975222 & 2.658147 & 2.658147 & 3.975222 & 3.986802 \\
8 & $\mathrm{H}$ & 4.606944 & 3.968609 & 2.625380 & 2.625380 & 3.968609 & 2.643207 & 1.210328 & 2.643207 & 3.990082 & 3.990082 & 2.638428 \\
9 & $\mathrm{H}$ & 3.934737 & 2.611663 & 1.211514 & 2.611663 & 3.934737 & 3.975222 & 2.658147 & 2.658147 & 3.975222 & 4.604555 & 3.986802 \\
10 & $\mathrm{H}$ & 3.968609 & 2.625380 & 2.625380 & 3.968609 & 4.606944 & 3.990082 & 2.643207 & 1.210328 & 2.643207 & 3.990082 & 2.638428 \\
11 & $\mathrm{H}$ & 2.625380 & 3.968609 & 4.606944 & 3.968609 & 2.625380 & 2.643207 & 3.990082 & 3.990082 & 2.643207 & 1.210328 & 2.638428 \\
\hline
\end{tabular}


24-3

\begin{tabular}{|ccccccccccccc}
\hline & & B1 & B2 & B3 & B4 & B5 & B6 & B7 & B8 & B9 & B10 & B11 \\
1 & $\mathrm{H}$ & 2.639372 & 2.639424 & 4.173804 & 5.262305 & 4.006550 & 2.639372 & 4.006550 & 5.262305 & 4.173804 & 2.639424 & 1.204824 \\
2 & $\mathrm{H}$ & 3.735131 & 2.794026 & 4.000255 & 4.469502 & 4.060395 & 1.208857 & 2.508983 & 3.826008 & 2.720138 & 2.757794 & 2.533283 \\
3 & $\mathrm{H}$ & 2.793907 & 3.734930 & 4.000295 & 3.825599 & 2.509359 & 2.757818 & 4.059570 & 4.468869 & 2.719060 & 1.208820 & 2.533468 \\
4 & $\mathrm{H}$ & 4.025113 & 2.512112 & 2.992308 & 3.783033 & 4.227901 & 2.511466 & 1.215223 & 2.391833 & 2.992426 & 4.024514 & 3.752793 \\
5 & $\mathrm{H}$ & 4.655719 & 4.012535 & 2.932652 & 2.513513 & 3.905509 & 4.012641 & 2.451775 & 1.215040 & 2.933107 & 4.655500 & 5.190565 \\
6 & $\mathrm{H}$ & 4.012641 & 4.655500 & 2.933107 & 1.215040 & 2.451775 & 4.655719 & 3.905509 & 2.513513 & 2.932652 & 4.012535 & 5.190565 \\
7 & $\mathrm{H}$ & 2.511466 & 4.024514 & 2.992426 & 2.391833 & 1.215223 & 4.025113 & 4.227901 & 3.783033 & 2.992308 & 2.512112 & 3.752793 \\
8 & $\mathrm{H}$ & 1.208857 & 2.757794 & 2.720138 & 3.826008 & 2.508983 & 3.735131 & 4.060395 & 4.469502 & 4.000255 & 2.794026 & 2.533283 \\
9 & $\mathrm{H}$ & 2.757818 & 1.208820 & 2.719060 & 4.468869 & 4.059570 & 2.793907 & 2.509359 & 3.825599 & 4.000295 & 3.734930 & 2.533468 \\
10 & $\mathrm{H}$ & 2.635720 & 2.634927 & 1.210657 & 2.700286 & 2.895918 & 3.961435 & 2.895946 & 2.700051 & 3.651395 & 3.961731 & 3.899882 \\
11 & $\mathrm{H}$ & 3.961435 & 3.961731 & 3.651395 & 2.700051 & 2.895946 & 2.635720 & 2.895918 & 2.700286 & 1.210657 & 2.634927 & 3.899882 \\
\hline
\end{tabular}

\section{4-4}

\begin{tabular}{|cccccccccccccc}
\hline & & $\mathrm{B} 1$ & $\mathrm{~B} 2$ & $\mathrm{~B} 3$ & $\mathrm{~B} 4$ & $\mathrm{~B} 5$ & $\mathrm{~B} 6$ & $\mathrm{~B} 7$ & B8 & B9 & B10 & B11 \\
1 & $\mathrm{H}$ & 1.376145 & 3.737538 & 2.542159 & 3.371677 & 4.063673 & 4.434741 & 3.871685 & 2.570177 & 1.274651 & 2.614518 & 3.786545 \\
2 & $\mathrm{H}$ & 2.302186 & 2.677241 & 2.773054 & 4.038670 & 4.848369 & 3.958669 & 3.936293 & 4.273422 & 2.938977 & 1.208448 & 2.500854 \\
3 & $\mathrm{H}$ & 2.499765 & 2.545189 & 1.212557 & 2.632078 & 4.364757 & 3.956286 & 4.807688 & 3.928309 & 2.991619 & 2.893489 & 4.069672 \\
4 & $\mathrm{H}$ & 3.839788 & 1.215458 & 2.497839 & 2.779008 & 3.930621 & 2.510508 & 3.920381 & 4.347111 & 3.807380 & 2.674347 & 2.692474 \\
5 & $\mathrm{H}$ & 5.281859 & 2.535319 & 4.066856 & 2.798273 & 2.546877 & 1.213292 & 2.739944 & 3.874002 & 4.365297 & 3.988181 & 2.645856 \\
6 & $\mathrm{H}$ & 5.437631 & 4.048910 & 4.550634 & 2.659247 & 1.209997 & 2.629847 & 2.669857 & 2.513897 & 3.981049 & 4.918736 & 3.963944 \\
7 & $\mathrm{H}$ & 5.083985 & 4.013784 & 4.892633 & 3.842093 & 2.520646 & 2.771314 & 1.217449 & 2.936736 & 3.751491 & 4.054941 & 2.594918 \\
8 & $\mathrm{H}$ & 3.927328 & 4.339987 & 3.860964 & 2.898872 & 2.412449 & 3.840339 & 3.046024 & 1.220701 & 2.481166 & 4.230872 & 4.165772 \\
9 & $\mathrm{H}$ & 3.965756 & 2.726556 & 2.509683 & 1.208833 & 2.532480 & 2.770629 & 3.801838 & 2.857659 & 3.209157 & 3.867675 & 2.594918 \\
10 & $\mathrm{H}$ & 4.244853 & 2.597951 & 3.949587 & 3.909698 & 3.869623 & 2.611220 & 2.583743 & 4.144461 & 3.782619 & 2.552359 & 3.932956 \\
11 & $\mathrm{H}$ & 1.198186 & 4.020384 & 2.702058 & 4.398545 & 5.521862 & 5.339092 & 5.165077 & 4.307197 & 2.698501 & 2.640497 & 4.396614 \\
\hline
\end{tabular}


Table S4. B/B and B/H Connectivities of $\mathrm{B}_{11} \mathrm{H}_{11^{4-}}$

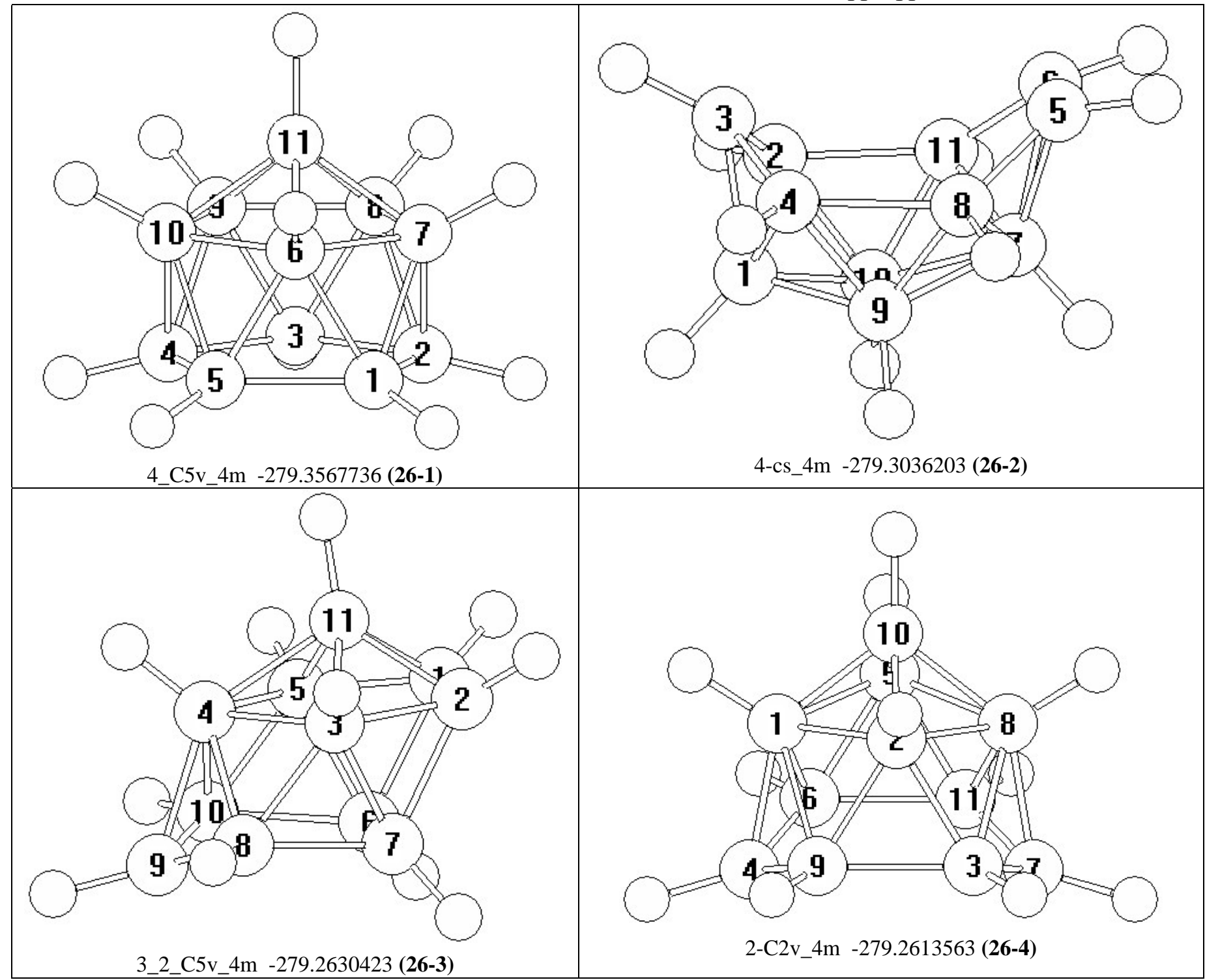

\section{B/B connectivities}

\section{6-1}

\begin{tabular}{|c|c|c|c|c|c|c|c|c|c|c|c|}
\hline & 1 & 2 & 3 & 4 & 5 & & 6 & 7 & 8 & 9 & 10 \\
\hline $1 \mathrm{~B}$ & 0.000000 & & & & & $6 \mathrm{~B}$ & 0.000000 & & & & \\
\hline $2 \mathrm{~B}$ & 1.791907 & 0.000000 & & & & $7 \mathrm{~B}$ & 1.795055 & 0.000000 & & & \\
\hline $3 \mathrm{~B}$ & 2.899366 & 1.791907 & 0.000000 & & & $8 \mathrm{~B}$ & 2.904461 & 1.795055 & 0.000000 & & \\
\hline $4 \mathrm{~B}$ & 2.899366 & 2.899366 & 1.791907 & 0.000000 & & $9 \mathrm{~B}$ & 2.904461 & 2.904461 & 1.795055 & 0.000000 & \\
\hline $5 \mathrm{~B}$ & 1.791907 & 2.899366 & 2.899366 & 1.791907 & 0.000000 & $10 \mathrm{~B}$ & 1.795055 & 2.904461 & 2.904461 & 1.795055 & 0.000000 \\
\hline $6 \mathrm{~B}$ & 1.822960 & 2.920223 & 3.426992 & 2.920223 & 1.822960 & $11 \mathrm{~B}$ & 1.803149 & 1.803149 & 1.803149 & 1.803149 & 1.803149 \\
\hline $7 \mathrm{~B}$ & 1.822960 & 1.822960 & 2.920223 & 3.426992 & 2.920223 & & & & & & \\
\hline $8 \mathrm{~B}$ & 2.920223 & 1.822960 & 1.822960 & 2.920223 & 3.426992 & & & & & & \\
\hline $9 \mathrm{~B}$ & 3.426992 & 2.920223 & 1.822960 & 1.822960 & 2.920223 & & & & & & \\
\hline $10 \mathrm{~B}$ & 2.920223 & 3.426992 & 2.920223 & 1.822960 & 1.822960 & & & & & & \\
\hline $11 \mathrm{~B}$ & 2.944450 & 2.944450 & 2.944450 & 2.944450 & 2.944450 & & & & & & \\
\hline
\end{tabular}


26-2

\begin{tabular}{|c|c|c|c|c|c|c|c|c|c|c|c|}
\hline & 1 & 2 & 3 & 4 & 5 & & 6 & 7 & 8 & 9 & 10 \\
\hline $1 \mathrm{~B}$ & 0.000000 & & & & & $6 \mathrm{~B}$ & 0.000000 & & & & \\
\hline $2 \mathrm{~B}$ & 1.809845 & 0.000000 & & & & $7 \mathrm{~B}$ & 1.938025 & 0.000000 & & & \\
\hline $3 \mathrm{~B}$ & 1.804666 & 1.684715 & 0.000000 & & & $8 \mathrm{~B}$ & 2.870629 & 1.948773 & 0.000000 & & \\
\hline $4 \mathrm{~B}$ & 1.809845 & 2.768574 & 1.684715 & 0.000000 & & $9 \mathrm{~B}$ & 3.494816 & 1.899656 & 1.774723 & 0.000000 & \\
\hline $5 \mathrm{~B}$ & 4.110680 & 3.900838 & 3.840857 & 3.263750 & 0.000000 & $10 \mathrm{~B}$ & 3.034076 & 1.899656 & 3.028077 & 1.824574 & 0.000000 \\
\hline $6 \mathrm{~B}$ & 4.110680 & 3.263750 & 3.840857 & 3.900838 & 1.648672 & $11 \mathrm{~B}$ & 1.673689 & 1.948773 & 3.299186 & 3.028077 & 1.774723 \\
\hline $7 \mathrm{~B}$ & 3.063579 & 3.080646 & 3.616533 & 3.080646 & 1.938025 & & & & & & \\
\hline $8 \mathrm{~B}$ & 3.079504 & 3.607930 & 3.169857 & 1.970562 & 1.673689 & & & & & & \\
\hline $9 \mathrm{~B}$ & 1.762892 & 2.877012 & 2.840971 & 1.796032 & 3.034076 & & & & & & \\
\hline $10 \mathrm{~B}$ & 1.762892 & 1.796032 & 2.840971 & 2.877012 & 3.494816 & & & & & & \\
\hline $11 \mathrm{~B}$ & 3.079504 & 1.970562 & 3.169857 & 3.607930 & 2.870629 & & & & & & \\
\hline
\end{tabular}

26-3

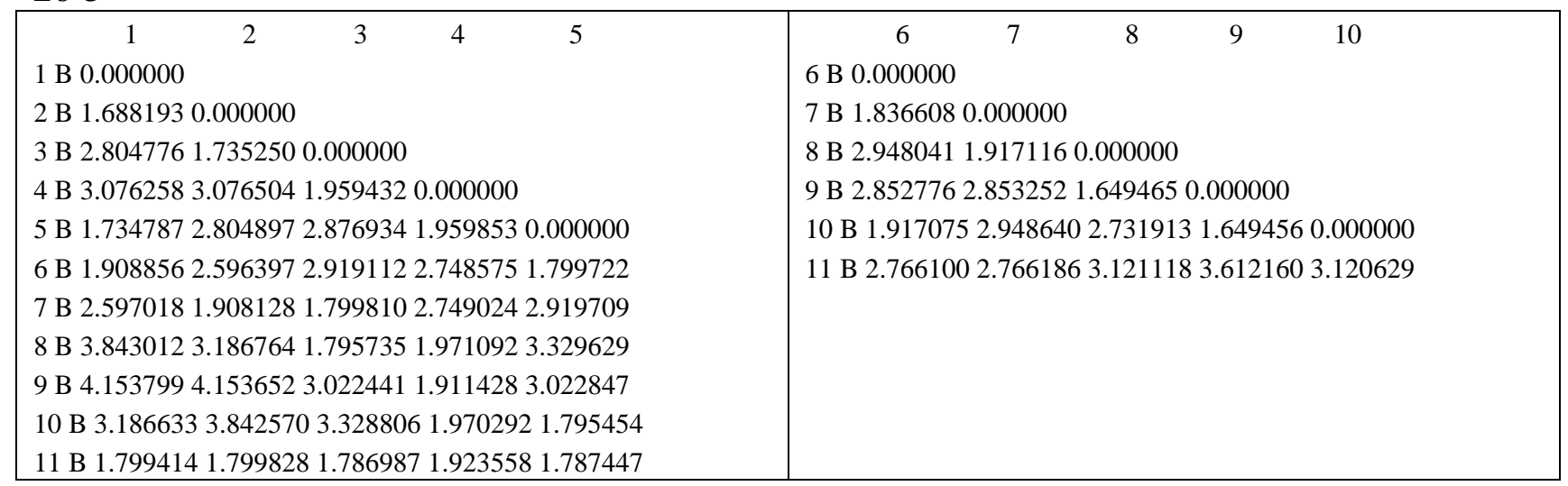

\section{6-4}

\begin{tabular}{|c|c|c|c|c|c|c|c|c|c|c|c|}
\hline & 1 & 2 & 3 & 4 & 5 & & 6 & 7 & 8 & 9 & 10 \\
\hline $1 \mathrm{~B}$ & 0.000000 & & & & & $6 \mathrm{~B}$ & 0.000000 & & & & \\
\hline $2 \mathrm{~B}$ & 1.967899 & 0.000000 & & & & $7 \mathrm{~B}$ & 3.023156 & 0.000000 & & & \\
\hline $3 \mathrm{~B}$ & 3.039130 & 1.835958 & 0.000000 & & & $8 \mathrm{~B}$ & 3.039130 & 1.851160 & 0.000000 & & \\
\hline $4 \mathrm{~B}$ & 1.851160 & 2.991618 & 3.023156 & 0.000000 & & $9 \mathrm{~B}$ & 2.822520 & 3.023675 & 3.039406 & 0.000000 & \\
\hline $5 \mathrm{~B}$ & 1.968682 & 2.762712 & 3.340720 & 2.993741 & 0.000000 & $10 \mathrm{~B}$ & 3.010879 & 3.405625 & 1.786210 & 3.012735 & 0.000000 \\
\hline $6 \mathrm{~B}$ & 2.011526 & 3.340720 & 3.385688 & 1.669351 & 1.835958 & $11 \mathrm{~B}$ & 1.871132 & 1.668900 & 2.014698 & 3.387125 & 3.012735 \\
\hline $7 \mathrm{~B}$ & 3.582940 & 2.993741 & 1.669351 & 3.396374 & 2.991618 & & & & & & \\
\hline $8 \mathrm{~B}$ & 2.770800 & 1.968682 & 2.011526 & 3.582940 & 1.967899 & & & & & & \\
\hline $9 \mathrm{~B}$ & 2.014698 & 1.835917 & 1.871132 & 1.668900 & 3.343131 & & & & & & \\
\hline $10 \mathrm{~B}$ & 1.786210 & 1.654930 & 3.010879 & 3.405625 & 1.654930 & & & & & & \\
\hline $11 \mathrm{~B}$ & 3.039406 & 3.343131 & 2.822520 & 3.023675 & 1.835917 & & & & & & \\
\hline
\end{tabular}




\section{B/H connectivities}

26-1

\begin{tabular}{|c|c|c|c|c|c|c|c|c|c|c|c|c|}
\hline & 1 & 2 & 3 & 4 & 5 & & 6 & 7 & 8 & 9 & 10 & 11 \\
\hline $12 \mathrm{H}$ & 4.091336 & 4.091336 & 2.718501 & 1.269797 & 2.718501 & $12 \mathrm{H}$ & 4.014853 & 4.680498 & 4.014853 & 2.598815 & 2.598815 & 3.974412 \\
\hline $13 \mathrm{H}$ & 2.718501 & 4.091336 & 4.091336 & 2.718501 & 1.269797 & $13 \mathrm{H}$ & 2.598815 & 4.014853 & 4.680498 & 4.014853 & 2.598815 & 3.974412 \\
\hline $14 \mathrm{H}$ & 1.269797 & 2.718501 & 4.091336 & 4.091336 & 2.718501 & $14 \mathrm{H}$ & 2.598815 & 2.598815 & 4.014853 & 4.680498 & 4.014853 & 3.974412 \\
\hline $15 \mathrm{H}$ & 2.718501 & 1.269797 & 2.718501 & 4.091336 & 4.091336 & $15 \mathrm{H}$ & 4.014853 & 2.598815 & 2.598815 & 4.014853 & 4.680498 & 3.974412 \\
\hline $16 \mathrm{H}$ & 4.091336 & 2.718501 & 1.269797 & 2.718501 & 4.091336 & $16 \mathrm{H}$ & 4.680498 & 4.014853 & 2.598815 & 2.598815 & 4.014853 & 3.974412 \\
\hline $17 \mathrm{H}$ & 4.675807 & 4.045617 & 2.733869 & 2.733869 & 4.045617 & $17 \mathrm{H}$ & 3.997124 & 3.997124 & 2.658646 & 1.250033 & 2.658646 & 2.630332 \\
\hline $18 \mathrm{H}$ & 4.045617 & 2.733869 & 2.733869 & 4.045617 & 4.675807 & $18 \mathrm{H}$ & 3.997124 & 2.658646 & 1.250033 & 2.658646 & 3.997124 & 2.630332 \\
\hline $19 \mathrm{H}$ & 4.045617 & 4.675807 & 4.045617 & 2.733869 & 2.733869 & $19 \mathrm{H}$ & 2.658646 & 3.997124 & 3.997124 & 2.658646 & 1.250033 & 2.630332 \\
\hline $20 \mathrm{H}$ & 2.733869 & 4.045617 & 4.675807 & 4.045617 & 2.733869 & $20 \mathrm{H}$ & 1.250033 & 2.658646 & 3.997124 & 3.997124 & 2.658646 & 2.630332 \\
\hline $21 \mathrm{H}$ & 2.733869 & 2.733869 & 4.045617 & 4.675807 & 4.045617 & $21 \mathrm{H}$ & 2.658646 & 1.250033 & 2.658646 & 3.997124 & 3.997124 & 2.630332 \\
\hline $22 \mathrm{H}$ & 4.058930 & 4.058930 & 4.058930 & 4.058930 & 4.058930 & $22 \mathrm{H}$ & 2.679362 & 2.679362 & 2.679362 & 2.679362 & 2.679362 & 1.242650 \\
\hline
\end{tabular}

26-2

\begin{tabular}{|c|c|c|c|c|c|c|c|c|c|c|c|c|}
\hline & 1 & 2 & 3 & 4 & 5 & & 6 & 7 & 8 & 9 & 10 & 11 \\
\hline $12 \mathrm{H}$ & 1.251887 & 2.748211 & 2.775226 & 2.748211 & 5.297965 & $12 \mathrm{H}$ & 5.297965 & 4.082717 & 4.128994 & 2.585748 & 2.585748 & 4.128994 \\
\hline $13 \mathrm{H}$ & 2.555669 & 3.961897 & 3.923405 & 2.696914 & 3.939717 & $13 \mathrm{H}$ & 4.531820 & 2.704353 & 2.572383 & 1.243071 & 2.663911 & 4.081029 \\
\hline $14 \mathrm{H}$ & 2.555669 & 2.696914 & 3.923405 & 3.961897 & 4.531820 & $14 \mathrm{H}$ & 3.939717 & 2.704353 & 4.081029 & 2.663911 & 1.243071 & 2.572383 \\
\hline $15 \mathrm{H}$ & 2.739972 & 2.596770 & 1.267133 & 2.596770 & 4.953653 & $15 \mathrm{H}$ & 4.953653 & 4.882239 & 4.284362 & 3.997034 & 3.997034 & 4.284362 \\
\hline $16 \mathrm{H}$ & 2.683538 & 1.257514 & 2.618067 & 3.920701 & 5.050552 & $16 \mathrm{H}$ & 4.158830 & 4.131309 & 4.858652 & 3.988660 & 2.611708 & 2.678229 \\
\hline $17 \mathrm{H}$ & 5.309125 & 4.326091 & 5.067188 & 5.181340 & 2.547917 & $17 \mathrm{H}$ & 1.284437 & 2.879990 & 3.998802 & 4.647938 & 4.030460 & 2.510140 \\
\hline $18 \mathrm{H}$ & 5.309125 & 5.181340 & 5.067188 & 4.326091 & 1.284437 & $18 \mathrm{H}$ & 2.547917 & 2.879990 & 2.510140 & 4.030460 & 4.647938 & 3.998802 \\
\hline $19 \mathrm{H}$ & 3.932126 & 4.111091 & 4.783595 & 4.111091 & 2.775842 & $19 \mathrm{H}$ & 2.775842 & 1.246389 & 2.817346 & 2.590161 & 2.590161 & 2.817346 \\
\hline $20 \mathrm{H}$ & 3.950930 & 4.788159 & 4.202822 & 2.708292 & 2.508895 & $20 \mathrm{H}$ & 3.947061 & 2.869023 & 1.264133 & 2.452074 & 4.035841 & 4.491979 \\
\hline $21 \mathrm{H}$ & 2.683538 & 3.920701 & 2.618067 & 1.257514 & 4.158830 & $21 \mathrm{H}$ & 5.050552 & 4.131309 & 2.678229 & 2.611708 & 3.988660 & 4.858652 \\
\hline $22 \mathrm{H}$ & 3.950930 & 2.708292 & 4.202822 & 4.788159 & 3.947061 & $22 \mathrm{H}$ & 2.508895 & 2.869023 & 4.491979 & 4.035841 & 2.452074 & 1.264133 \\
\hline
\end{tabular}

26-3

\begin{tabular}{|c|c|c|c|c|c|c|c|c|c|c|c|c|}
\hline & 1 & 2 & 3 & 4 & 5 & & 6 & 7 & 8 & 9 & 10 & 11 \\
\hline $12 \mathrm{H}$ & 2.715273 & 2.715359 & 2.673995 & 2.691456 & 2.675302 & $12 \mathrm{H}$ & 3.959927 & 3.959579 & 4.100601 & 4.567447 & 4.100574 & 1.243319 \\
\hline $13 \mathrm{H}$ & 5.379382 & 5.379337 & 4.083408 & 2.803367 & 4.083726 & $13 \mathrm{H}$ & 4.082118 & 4.082627 & 2.584036 & 1.283426 & 2.583879 & 4.681076 \\
\hline $14 \mathrm{H}$ & 4.951306 & 4.004012 & 2.439621 & 2.906951 & 4.517691 & $14 \mathrm{H}$ & 4.192195 & 2.889415 & 1.270868 & 2.557416 & 3.916810 & 4.016912 \\
\hline $15 \mathrm{H}$ & 4.003843 & 4.950868 & 4.516707 & 2.905997 & 2.439226 & $15 \mathrm{H}$ & 2.889536 & 4.192687 & 3.916608 & 2.557103 & 1.270723 & 4.016405 \\
\hline $16 \mathrm{H}$ & 2.624726 & 3.526793 & 4.119067 & 3.953415 & 2.739241 & $16 \mathrm{H}$ & 1.257697 & 2.761032 & 4.029000 & 3.758237 & 2.692366 & 3.903656 \\
\hline $17 \mathrm{H}$ & 3.528072 & 2.624558 & 2.739562 & 3.953601 & 4.119678 & $17 \mathrm{H}$ & 2.761284 & 1.257602 & 2.691638 & 3.758148 & 4.029455 & 3.903938 \\
\hline $18 \mathrm{H}$ & 4.038793 & 4.039420 & 2.805553 & 1.241149 & 2.805446 & $18 \mathrm{H}$ & 3.938019 & 3.938658 & 2.849393 & 2.643840 & 2.848044 & 2.576374 \\
\hline $19 \mathrm{H}$ & 3.842353 & 2.480047 & 1.241253 & 2.870296 & 4.027684 & $19 \mathrm{H}$ & 4.142455 & 2.790955 & 2.477469 & 3.912355 & 4.480518 & 2.632131 \\
\hline $20 \mathrm{H}$ & 2.673726 & 1.264521 & 2.519546 & 4.144064 & 3.975627 & $20 \mathrm{H}$ & 3.813722 & 2.916481 & 4.125141 & 5.298499 & 5.098603 & 2.682639 \\
\hline $21 \mathrm{H}$ & 1.264518 & 2.673955 & 3.975751 & 4.144114 & 2.519251 & $21 \mathrm{H}$ & 2.917105 & 3.814136 & 5.099124 & 5.298699 & 4.125062 & 2.682853 \\
\hline $22 \mathrm{H}$ & 2.479293 & 3.842559 & 4.027911 & 2.871149 & 1.241242 & $22 \mathrm{H}$ & 2.790702 & 4.142923 & 4.481548 & 3.912949 & 2.477250 & 2.633042 \\
\hline \multicolumn{13}{|l|}{$26-4$} \\
\hline & 1 & 2 & 3 & 4 & 5 & & 6 & 7 & 8 & 9 & 10 & 11 \\
\hline $12 \mathrm{H}$ & 2.744305 & 2.557917 & 4.097421 & 4.524282 & 2.557917 & $12 \mathrm{H}$ & 4.097421 & 4.524282 & 2.744305 & 4.099318 & 1.241459 & 4.099318 \\
\hline $13 \mathrm{H}$ & 4.094330 & 4.498047 & 3.991874 & 4.116788 & 2.488712 & $13 \mathrm{H}$ & 2.695551 & 2.582740 & 2.896984 & 4.647743 & 3.880481 & 1.266586 \\
\hline $14 \mathrm{H}$ & 2.896984 & 2.488712 & 2.695551 & 2.582740 & 4.498047 & $14 \mathrm{H}$ & 3.991874 & 4.116788 & 4.094330 & 1.266586 & 3.880481 & 4.647743 \\
\hline $15 \mathrm{H}$ & 2.682941 & 4.033399 & 4.190564 & 1.278496 & 4.035246 & $15 \mathrm{H}$ & 2.574859 & 4.633586 & 4.835313 & 2.575364 & 4.427580 & 4.190133 \\
\hline $16 \mathrm{H}$ & 2.891918 & 4.494823 & 4.646494 & 2.581770 & 2.489044 & $16 \mathrm{H}$ & 1.266552 & 4.117270 & 4.094912 & 3.990911 & 3.877963 & 2.696555 \\
\hline $17 \mathrm{H}$ & 3.876831 & 2.835120 & 2.890796 & 4.825648 & 2.833777 & $17 \mathrm{H}$ & 4.179727 & 2.599679 & 1.243240 & 4.179974 & 2.476073 & 2.893748 \\
\hline $18 \mathrm{H}$ & 2.944643 & 1.246724 & 2.617286 & 4.004980 & 3.890278 & $18 \mathrm{H}$ & 4.550728 & 4.007917 & 2.945703 & 2.616371 & 2.473880 & 4.553492 \\
\hline $19 \mathrm{H}$ & 1.243240 & 2.833777 & 4.179727 & 2.599679 & 2.835120 & $19 \mathrm{H}$ & 2.890796 & 4.825648 & 3.876831 & 2.893748 & 2.476073 & 4.179974 \\
\hline $20 \mathrm{H}$ & 2.945703 & 3.890278 & 4.550728 & 4.007917 & 1.246724 & $20 \mathrm{H}$ & 2.617286 & 4.004980 & 2.944643 & 4.553492 & 2.473880 & 2.616371 \\
\hline $21 \mathrm{H}$ & 4.094912 & 2.489044 & 1.266552 & 4.117270 & 4.494823 & $21 \mathrm{H}$ & 4.646494 & 2.581770 & 2.891918 & 2.696555 & 3.877963 & 3.990911 \\
\hline $22 \mathrm{H}$ & 4.835313 & 4.035246 & 2.574859 & 4.633586 & 4.033399 & $22 \mathrm{H}$ & 4.190564 & 1.278496 & 2.682941 & 4.190133 & 4.427580 & 2.575364 \\
\hline
\end{tabular}


Table S4. B/B and B/H Connectivities of $\mathrm{B}_{11} \mathrm{H}_{11}{ }^{6-}$

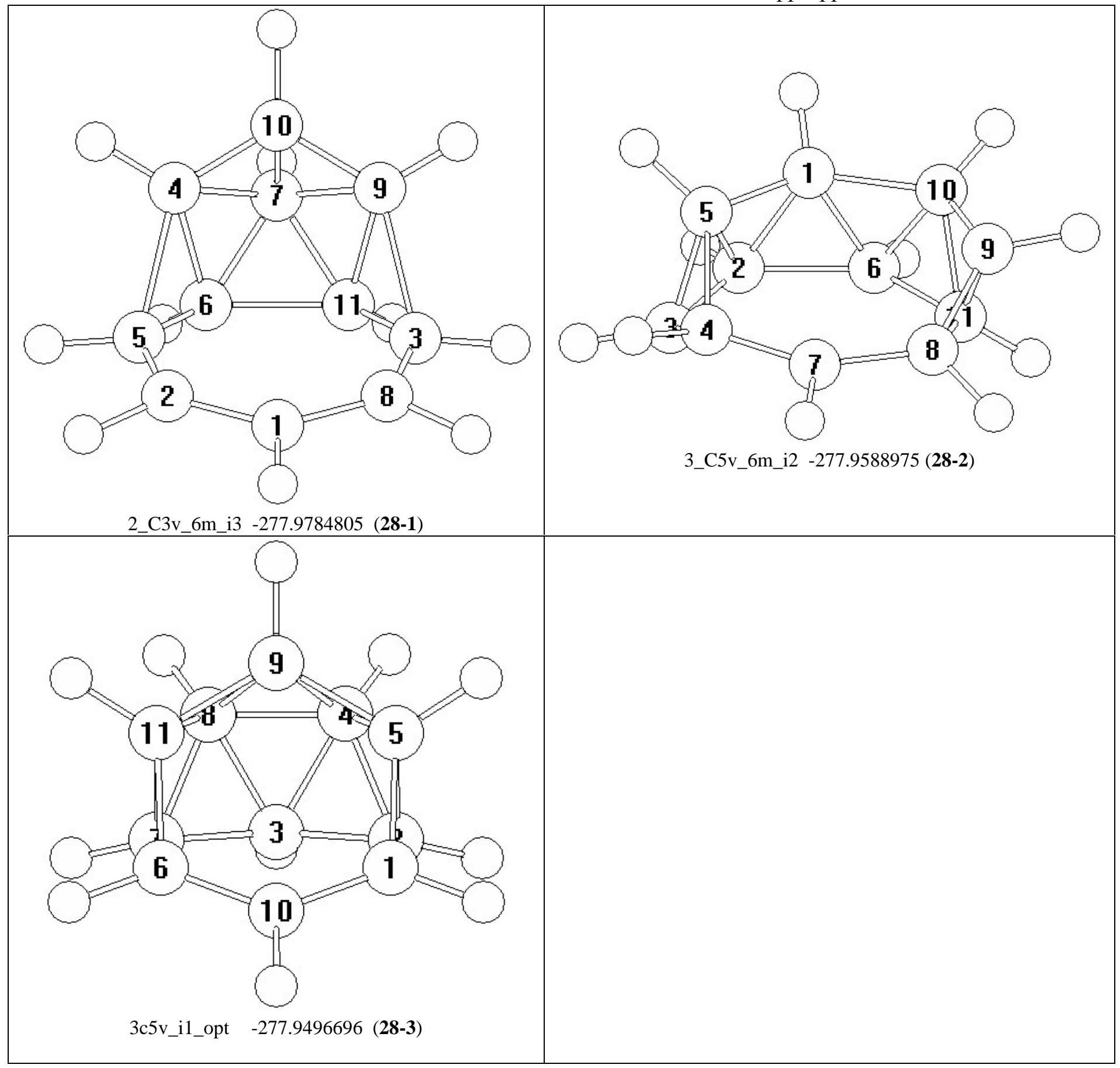




\section{B-B connectivities}

\section{8-1}

\begin{tabular}{|c|c|c|c|c|c|c|c|c|c|c|}
\hline & 1 & 2 & 3 & 4 & 5 & 6 & 7 & 8 & 9 & 10 \\
\hline $1 \mathrm{~B}$ & 0.000000 & & & & & & & & & \\
\hline $2 \mathrm{~B}$ & 1.707543 & 0.000000 & & & & & & & & \\
\hline $3 \mathrm{~B}$ & 3.133592 & 3.810600 & 0.000000 & & & & & & & \\
\hline $4 \mathrm{~B}$ & 4.532210 & 3.564751 & 3.948994 & 0.000000 & & & & & & \\
\hline $5 \mathrm{~B}$ & 3.133979 & 1.741768 & 3.800728 & 2.202905 & 0.000000 & & & & & \\
\hline $6 \mathrm{~B}$ & 4.114592 & 3.252860 & 3.253574 & 1.846341 & 1.784227 & 0.000000 & & & & \\
\hline $7 \mathrm{~B}$ & 5.045424 & 4.520319 & 3.242601 & 1.801158 & 3.239438 & 1.810402 & 0.000000 & & & \\
\hline $8 \mathrm{~B}$ & 1.707217 & 3.022406 & 1.741457 & 4.609456 & 3.810570 & 4.057850 & 4.521834 & 0.000000 & & \\
\hline $9 \mathrm{~B}$ & 4.534243 & 4.609431 & 2.208823 & 2.820507 & 3.946779 & 2.983132 & 1.800476 & 3.568904 & 0.000000 & \\
\hline $10 \mathrm{~B}$ & 4.755609 & 4.352251 & 3.480384 & 1.701968 & 3.476424 & 2.913494 & 1.811089 & 4.354427 & 1.701166 & 0.000000 \\
\hline $11 \mathrm{~B}$ & 4.114592 & 4.058032 & 1.784150 & 2.983948 & 3.253438 & 1.947516 & 1.811447 & 3.252739 & 1.846102 & 2.913519 \\
\hline
\end{tabular}

28-2

\begin{tabular}{|c|c|c|c|c|c|c|c|c|c|c|}
\hline & 1 & 2 & 3 & 4 & 5 & 6 & 7 & 8 & 9 & 10 \\
\hline $1 \mathrm{~B}$ & 0.000000 & & & & & & & & & \\
\hline $2 \mathrm{~B}$ & 1.857615 & 0.000000 & & & & & & & & \\
\hline $3 \mathrm{~B}$ & 2.980754 & 1.714330 & 0.000000 & & & & & & & \\
\hline $4 \mathrm{~B}$ & 3.341651 & 2.952346 & 1.730782 & 0.000000 & & & & & & \\
\hline $5 \mathrm{~B}$ & 1.748725 & 1.834269 & 1.794362 & 2.073569 & 0.000000 & & & & & \\
\hline $6 \mathrm{~B}$ & 1.841098 & 1.933473 & 3.242218 & 3.794216 & 2.993516 & 0.000000 & & & & \\
\hline $7 \mathrm{~B}$ & 3.800940 & 3.831524 & 3.114955 & 1.735529 & 3.238641 & 3.800519 & 0.000000 & & & \\
\hline $8 \mathrm{~B}$ & 3.794209 & 4.229255 & 4.178917 & 3.230915 & 4.023845 & 3.341010 & 1.735430 & 0.000000 & & \\
\hline $9 \mathrm{~B}$ & 3.242321 & 4.324231 & 4.843398 & 4.179212 & 4.129443 & 2.980928 & 3.115156 & 1.731054 & 0.000000 & \\
\hline $10 \mathrm{~B}$ & 1.933468 & 3.284106 & 4.324102 & 4.229598 & 3.382500 & 1.857783 & 3.831642 & 2.952203 & 1.714338 & 0.000000 \\
\hline $11 \mathrm{~B}$ & 2.993248 & 3.382356 & 4.128880 & 4.022910 & 3.951004 & 1.748874 & 3.237093 & 2.071844 & 1.794012 & 1.834267 \\
\hline
\end{tabular}

\section{8-3}

\begin{tabular}{|c|c|c|c|c|c|c|c|c|c|c|}
\hline & 1 & 2 & 3 & 4 & 5 & 6 & 7 & 8 & 9 & 10 \\
\hline $1 \mathrm{~B}$ & 0.000000 & & & & & & & & & \\
\hline $2 \mathrm{~B}$ & 1.869268 & 0.000000 & & & & & & & & \\
\hline $3 \mathrm{~B}$ & 3.181934 & 1.821587 & 0.000000 & & & & & & & \\
\hline $4 \mathrm{~B}$ & 3.094798 & 1.823990 & 1.845569 & 0.000000 & & & & & & \\
\hline $5 \mathrm{~B}$ & 1.867541 & 1.866270 & 2.972620 & 1.822914 & 0.000000 & & & & & \\
\hline $6 \mathrm{~B}$ & 3.002714 & 3.587465 & 3.183044 & 3.866208 & 3.587997 & 0.000000 & & & & \\
\hline $7 \mathrm{~B}$ & 3.583787 & 3.119297 & 1.821983 & 2.982364 & 3.635363 & 1.867235 & 0.000000 & & & \\
\hline $8 \mathrm{~B}$ & 3.862951 & 2.982299 & 1.846012 & 1.785243 & 2.981575 & 3.093710 & 1.823255 & 0.000000 & & \\
\hline $9 \mathrm{~B}$ & 3.179798 & 2.973260 & 2.957919 & 1.845469 & 1.821543 & 3.183131 & 2.973899 & 1.845395 & 0.000000 & \\
\hline $10 \mathrm{~B}$ & 1.718806 & 3.055679 & 3.545137 & 3.973040 & 3.054468 & 1.718445 & 3.052402 & 3.970574 & 3.542485 & 0.000000 \\
\hline $11 \mathrm{~B}$ & 3.582575 & 3.635543 & 2.973910 & 2.982533 & 3.119695 & 1.867021 & 1.867503 & 1.823032 & 1.822293 & 3.050284 \\
\hline
\end{tabular}




\section{B-H connectivities}

\begin{tabular}{|c|c|c|c|c|c|c|c|c|c|c|c|}
\hline & B1 & B2 & B3 & B4 & B5 & B6 & B7 & B8 & B9 & B10 & B11 \\
\hline $1 \mathrm{H}$ & 2.666752 & 4.258951 & 2.641329 & 5.983830 & 5.218142 & 5.424988 & 5.755318 & 1.426362 & 4.560515 & 5.531514 & 4.365192 \\
\hline $2 \mathrm{H}$ & 5.900219 & 5.495590 & 4.641200 & 2.645291 & 4.636754 & 4.119846 & 2.802613 & 5.498213 & 2.644406 & 1.327919 & 4.119932 \\
\hline $3 \mathrm{H}$ & 2.667474 & 1.426817 & 5.218542 & 4.555932 & 2.642093 & 4.365562 & 5.753681 & 4.259329 & 5.983905 & 5.529158 & 5.425634 \\
\hline $4 \mathrm{H}$ & 4.223231 & 5.106152 & 1.346571 & 5.095342 & 5.112622 & 4.354817 & 4.054571 & 2.593826 & 2.813356 & 4.388829 & 2.549568 \\
\hline $5 \mathrm{H}$ & 6.291994 & 5.703841 & 4.271701 & 2.746512 & 4.269373 & 2.657564 & 1.297012 & 5.704940 & 2.744722 & 2.797065 & 2.658212 \\
\hline $6 \mathrm{H}$ & 5.603358 & 4.395548 & 5.241726 & 1.298875 & 2.925270 & 2.739849 & 2.703536 & 5.861926 & 3.962770 & 2.612102 & 4.145978 \\
\hline $7 \mathrm{H}$ & 5.219891 & 4.208583 & 4.344878 & 2.702156 & 2.619073 & 1.315502 & 2.508797 & 5.221875 & 4.027076 & 3.937886 & 2.809265 \\
\hline $8 \mathrm{H}$ & 4.222800 & 2.593269 & 5.112263 & 2.808532 & 1.346556 & 2.549454 & 4.051582 & 5.105457 & 5.093286 & 4.385787 & 4.354645 \\
\hline $9 \mathrm{H}$ & 5.220793 & 5.222890 & 2.618995 & 4.027633 & 4.345247 & 2.808580 & 2.508950 & 4.209155 & 2.701325 & 3.937396 & 1.315695 \\
\hline $10 \mathrm{H}$ & 1.433023 & 2.691020 & 4.356662 & 5.876311 & 4.357269 & 5.502079 & 6.475185 & 2.690564 & 5.878557 & 6.081172 & 5.502102 \\
\hline $11 \mathrm{H}$ & 5.604661 & 5.861476 & 2.929821 & 3.963769 & 5.239455 & 4.145245 & 2.704033 & 4.398830 & 1.298719 & 2.612258 & 2.739227 \\
\hline
\end{tabular}

\section{8-2}

\begin{tabular}{|c|c|c|c|c|c|c|c|c|c|c|c|}
\hline & B1 & B2 & B3 & B4 & B5 & B6 & B7 & B8 & B9 & B10 & B11 \\
\hline $1 \mathrm{H}$ & 2.746861 & 2.649178 & 4.194467 & 5.028566 & 4.047214 & 1.326692 & 5.089337 & 4.501515 & 3.960840 & 2.669824 & 2.594228 \\
\hline $2 \mathrm{H}$ & 2.741993 & 4.190207 & 5.465516 & 5.512321 & 4.407584 & 2.688460 & 5.146443 & 4.131294 & 2.616133 & 1.329057 & 2.796528 \\
\hline $3 \mathrm{H}$ & 5.190406 & 5.150224 & 4.156790 & 2.613135 & 4.449166 & 5.189404 & 1.420048 & 2.613550 & 4.158199 & 5.151166 & 4.447179 \\
\hline $4 \mathrm{H}$ & 5.137230 & 5.477721 & 5.334823 & 4.315320 & 5.365074 & 4.469482 & 2.632246 & 1.372161 & 2.593979 & 4.075782 & 2.926128 \\
\hline $5 \mathrm{H}$ & 4.470230 & 4.076005 & 2.593807 & 1.371975 & 2.927776 & 5.137166 & 2.632091 & 4.315058 & 5.334823 & 5.477966 & 5.363839 \\
\hline $6 \mathrm{H}$ & 2.609392 & 2.813474 & 2.667373 & 2.958771 & 1.322745 & 4.148075 & 4.312065 & 5.217419 & 5.208308 & 4.375361 & 5.209296 \\
\hline $7 \mathrm{H}$ & 1.326604 & 2.669444 & 3.960515 & 4.502182 & 2.593564 & 2.746766 & 5.089813 & 5.028575 & 4.194566 & 2.649197 & 4.047077 \\
\hline $8 \mathrm{H}$ & 2.688332 & 1.328925 & 2.615996 & 4.131292 & 2.796152 & 2.741967 & 5.146145 & 5.511791 & 5.465593 & 4.190234 & 4.407564 \\
\hline $9 \mathrm{H}$ & 4.068419 & 2.633784 & 1.357636 & 2.691409 & 2.721217 & 4.418138 & 4.261222 & 5.484946 & 6.196849 & 5.602638 & 5.441353 \\
\hline $10 \mathrm{H}$ & 4.418123 & 5.602842 & 6.196948 & 5.485284 & 5.441761 & 4.068767 & 4.261561 & 2.692022 & 1.357686 & 2.633746 & 2.721580 \\
\hline $11 \mathrm{H}$ & 4.148164 & 4.375464 & 5.207790 & 5.216199 & 5.209490 & 2.609705 & 4.309883 & 2.956334 & 2.666789 & 2.813735 & 1.322879 \\
\hline
\end{tabular}

\section{8-3}

\begin{tabular}{|c|c|c|c|c|c|c|c|c|c|c|c|}
\hline & B1 & B2 & B3 & B4 & B5 & B6 & B7 & B8 & B9 & B10 & B11 \\
\hline $1 \mathrm{H}$ & 4.261195 & 4.931113 & 4.340531 & 5.165610 & 4.932573 & 1.378715 & 2.722023 & 4.123085 & 4.342183 & 2.712024 & 2.723336 \\
\hline $2 \mathrm{H}$ & 2.777537 & 4.357384 & 4.955394 & 5.447663 & 4.350626 & 2.782904 & 4.358430 & 5.447080 & 4.944775 & 1.503588 & 4.350599 \\
\hline $3 \mathrm{H}$ & 4.882174 & 4.302831 & 2.713911 & 4.044215 & 4.936803 & 2.782312 & 1.337440 & 2.604460 & 4.080353 & 4.188054 & 2.765810 \\
\hline $4 \mathrm{H}$ & 5.147234 & 4.093686 & 2.768575 & 2.638812 & 4.093057 & 4.200992 & 2.749694 & 1.304887 & 2.768521 & 5.248191 & 2.749845 \\
\hline $5 \mathrm{H}$ & 4.203891 & 2.751447 & 2.769041 & 1.305420 & 2.750196 & 5.150699 & 4.094269 & 2.639801 & 2.768872 & 5.251631 & 4.094446 \\
\hline $6 \mathrm{H}$ & 2.783753 & 2.765902 & 4.080384 & 2.605733 & 1.337450 & 4.885724 & 4.937378 & 4.044735 & 2.713535 & 4.189477 & 4.303030 \\
\hline $7 \mathrm{H}$ & 1.380020 & 2.724149 & 4.340720 & 4.125277 & 2.723403 & 4.261868 & 4.928788 & 5.163948 & 4.339445 & 2.712666 & 4.928076 \\
\hline $8 \mathrm{H}$ & 2.783952 & 1.335822 & 2.712544 & 2.606144 & 2.764478 & 4.883417 & 4.301211 & 4.044613 & 4.079959 & 4.189201 & 4.936181 \\
\hline $9 \mathrm{H}$ & 4.350221 & 2.736407 & 1.326445 & 2.669946 & 4.104874 & 4.349433 & 2.735128 & 2.669914 & 4.074744 & 4.771024 & 4.105346 \\
\hline $10 \mathrm{H}$ & 4.346860 & 4.105814 & 4.074743 & 2.670542 & 2.736208 & 4.347864 & 4.104385 & 2.668968 & 1.325547 & 4.766252 & 2.733786 \\
\hline $11 \mathrm{H}$ & 4.880279 & 4.937428 & 4.081631 & 4.044832 & 4.302689 & 2.782288 & 2.767412 & 2.605411 & 2.713646 & 4.185095 & 1.337550 \\
\hline
\end{tabular}


Figure S1. Lowest lying molecular orbitals for isomer $24-1\left(C_{2 v}\right)$ of $\mathrm{B}_{11} \mathrm{H}_{11}{ }^{2-}$.

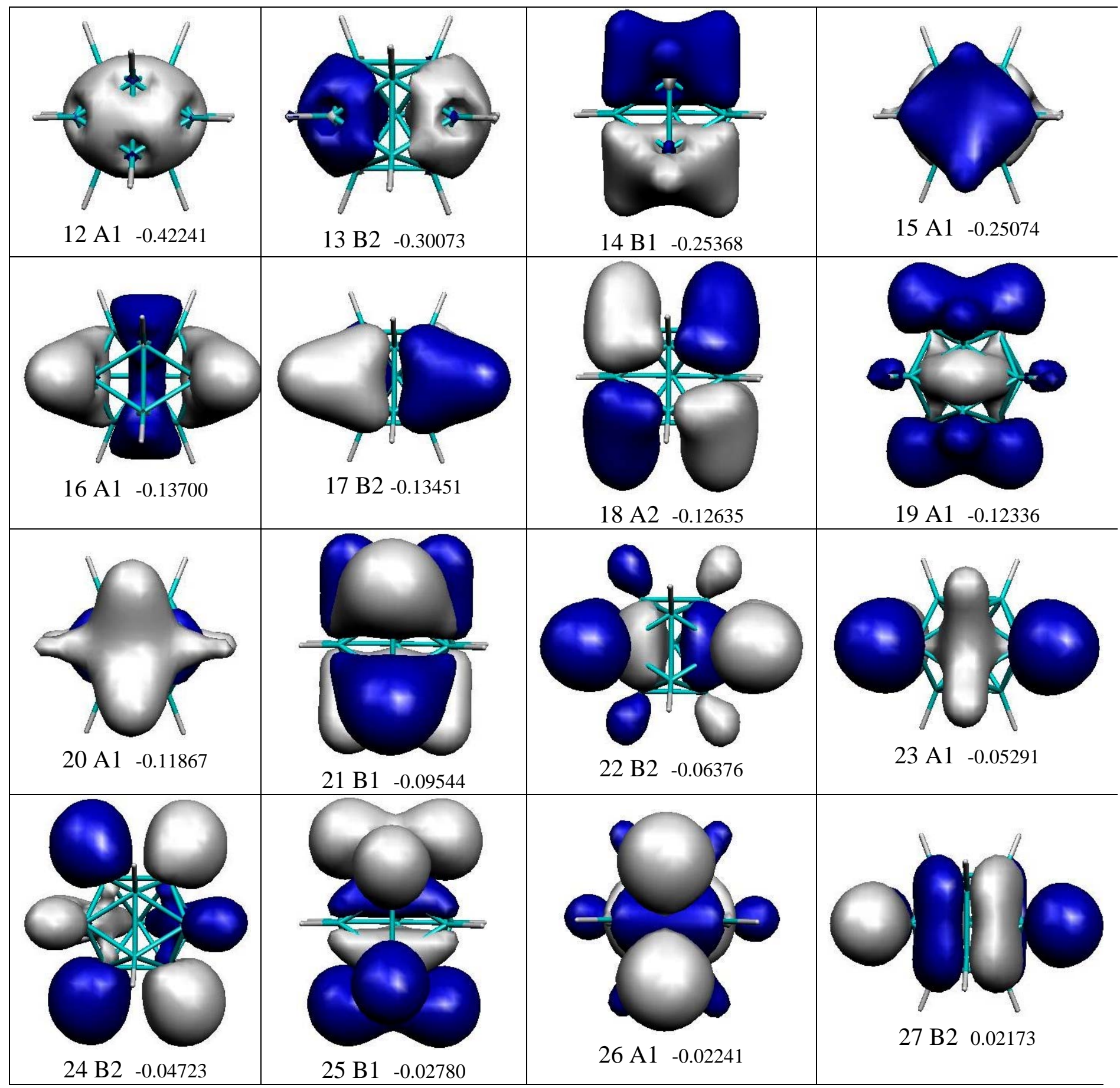




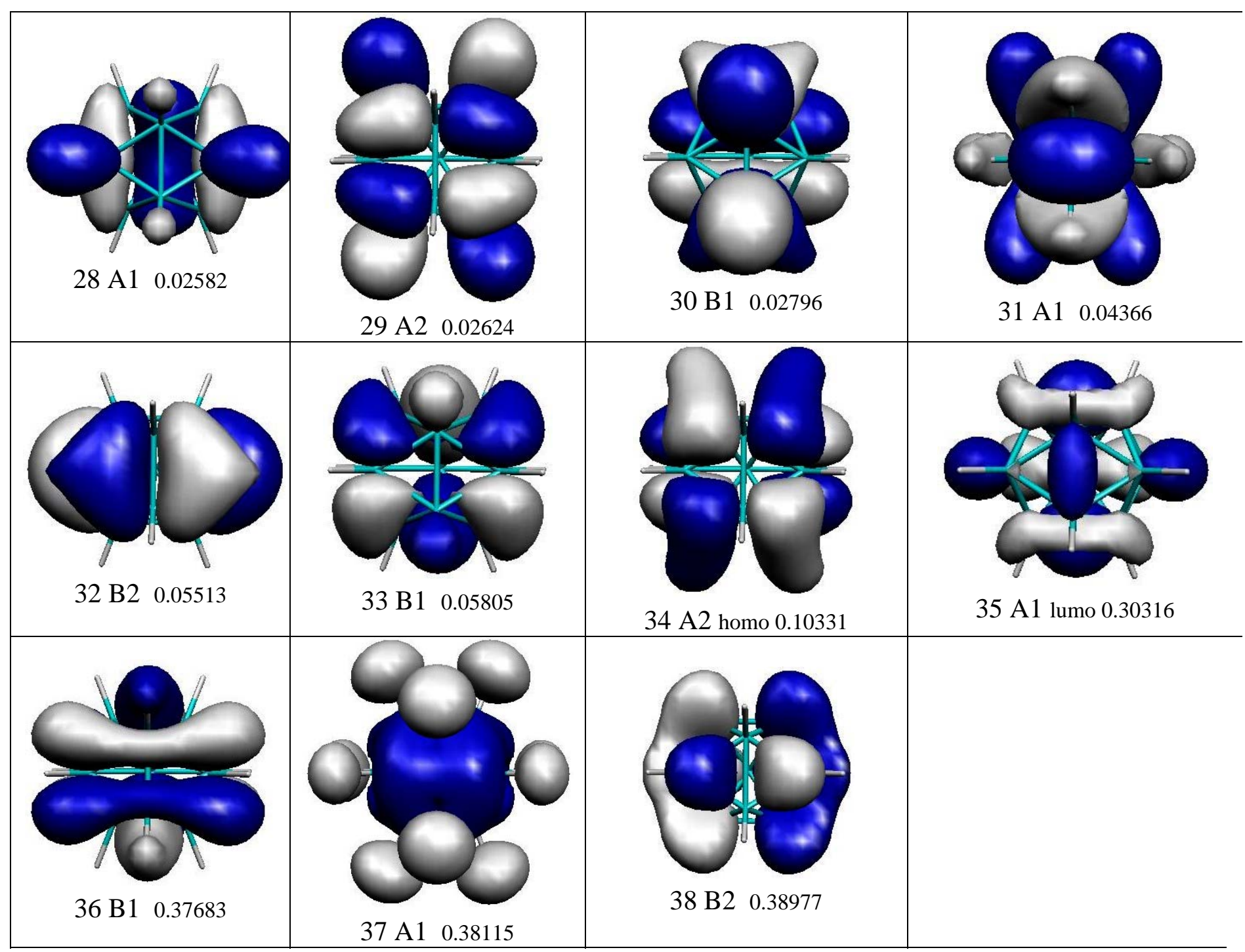


Figure S2. Lowest lying molecular orbitals for isomer $\mathbf{2 4 - 3}$ of $\mathrm{B}_{11} \mathrm{H}_{11}{ }^{2-}$.
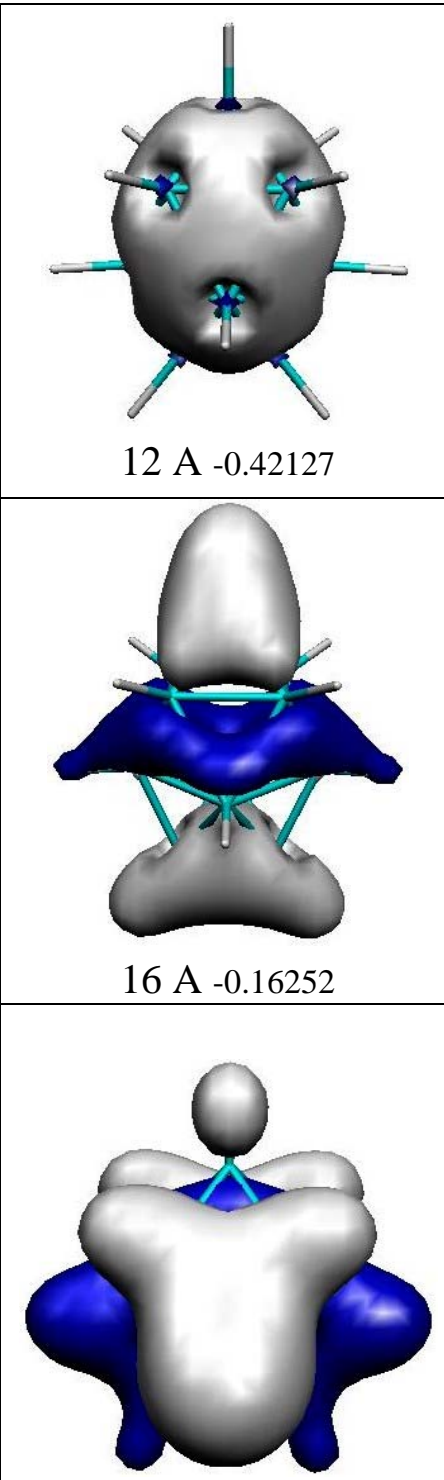

20 A -0.10625

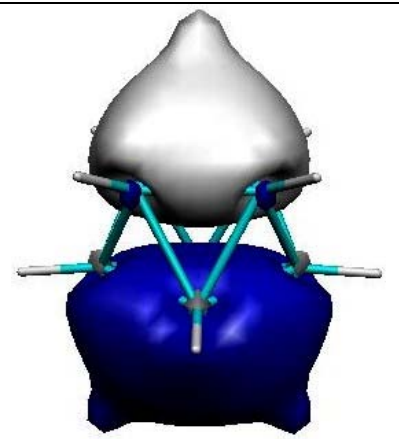

13 A -0.31106

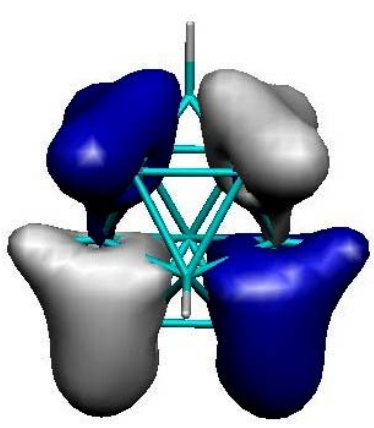

17 B -0.14106

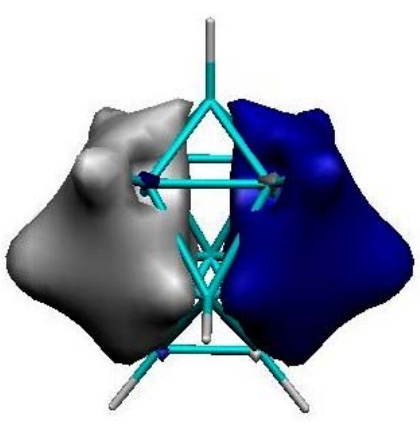

14 B -0.25612

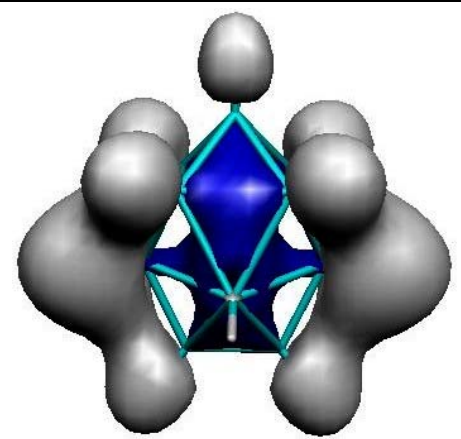

18 A -0.12677

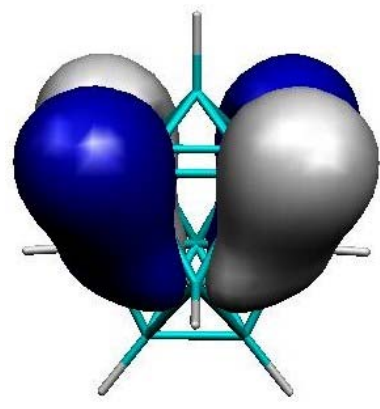

21 A -0.09787

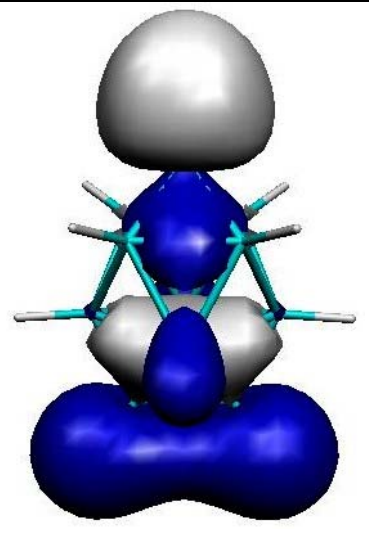

22 A -0.07883

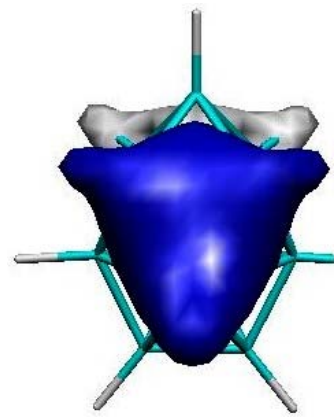

15 B -0.22521

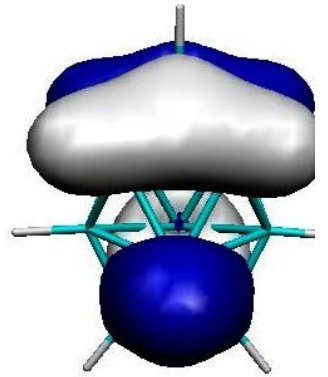

19 B -0.11356

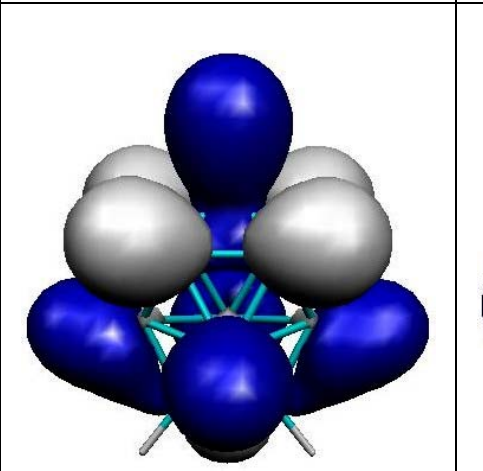

24 A -0.04528

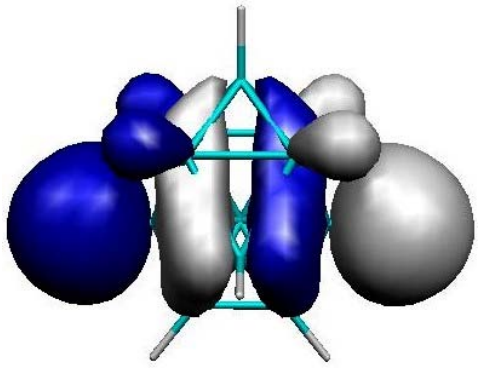

25 B -0.02873

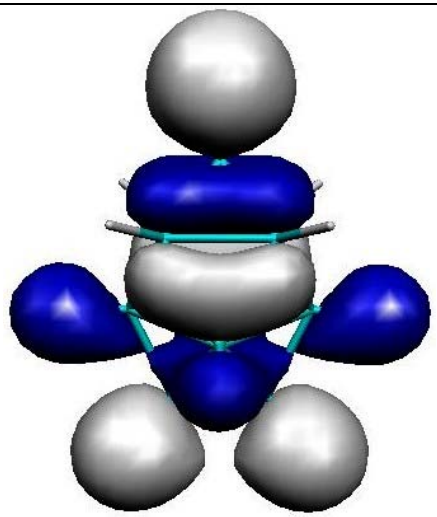

26 A -0.00733

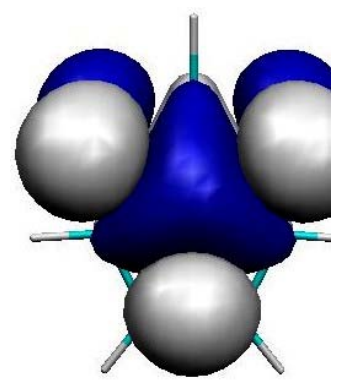

27 B 0.00131

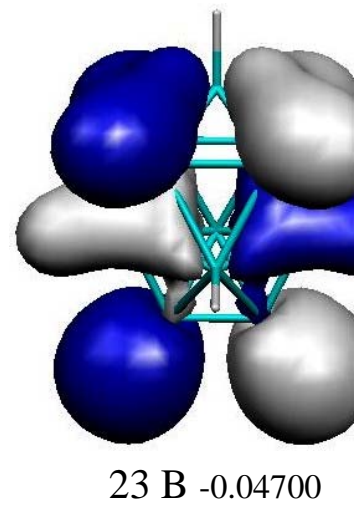




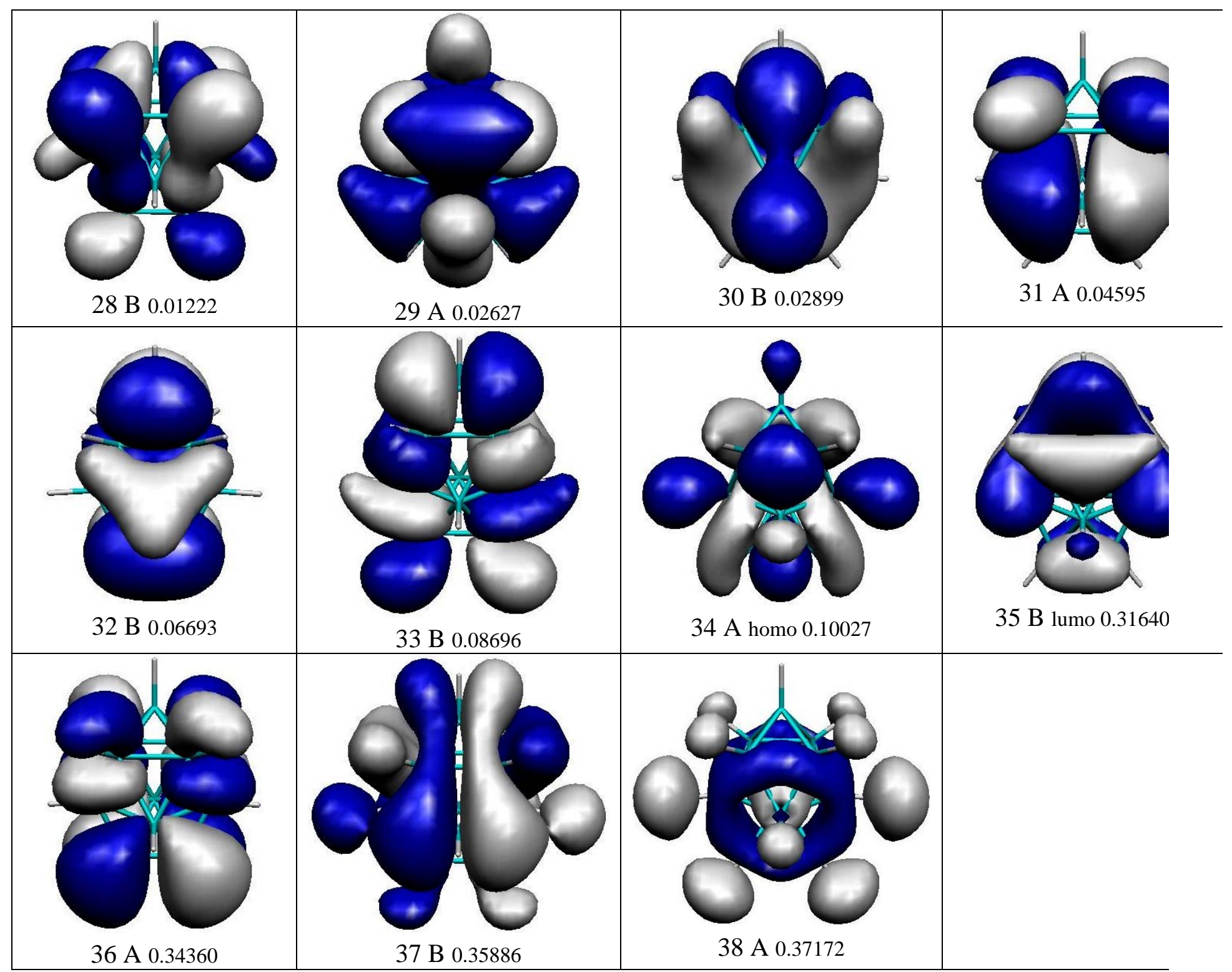

